\title{
Flatness of CR Submanifolds in a Sphere
}

\author{
Shanyu Ji and Yuan Yuan \\ November 11, 2018 \\ Dedicated to Professor Yang, Lo in the Occasion of his 70th Birthday
}

\section{Introduction}

The Cartan-Janet theorem asserted that for any analytic Riemannian manifold $\left(M^{n}, g\right)$, there exist local isometric embeddings of $M^{n}$ into Euclidean space $\mathbb{E}^{N}$ as $N$ is sufficiently large. The CR analogue of Cartan-Janet theorem is not true in general. In fact, Forstneric [F086] and Faran [Fa88] proved the existence of real analytic strictly pseudoconvex hypersurfaces $M^{2 n+1} \subset \mathbb{C}^{n+1}$ which do not admit any germ of holomorphic mapping taking $M$ into sphere $\partial \mathbb{B}^{N+1}$ for any $N$.

There are recent progress on $\mathrm{CR}$ submanifolds in sphere $\partial \mathbb{B}^{N+1}$. Zaitsev [Za08] constructed explicit examples for the Forstneric and Faran phenomenon above. Ebenfelt, Huang and Zaitsev [EHZ04] proved rigidity of CR embeddings of general $M^{2 n+1}$ into spheres with CR co-dimension $<\frac{n}{2}$, which generalizes a result of Webster that was for the case of codimension 1 We79]. S.-Y. Kim and J.-W. Oh [KO06] gave a necessary and sufficient condition for local embeddability into a sphere $\partial \mathbb{B}^{N+1}$ of a generic strictly pseudoconvex psuedohermitian CR manifold $\left(M^{2 n+1}, \theta\right)$ in terms of its Chern-Moser curvature tensors and their derivatives.

In Euclidean geometry, for a real submanifold $M^{n} \subset \mathbb{E}^{n+a}, M$ is a piece of $\mathbb{E}^{n}$ if and only if its second fundamental form $I I_{M} \equiv 0$. In projective geometry, for a complex submanifold $M^{n} \subset \mathbb{C P}^{n+a}, M$ is a piece of $\mathbb{C P}^{n}$ if and only if its projective second fundamental form $I I_{M} \equiv 0$ (c.f. [L03], p.81). In CR geometry, we prove the CR analogue of this fact in this paper as follows:

Theorem 1.1 Let $H: M^{\prime} \rightarrow \partial \mathbb{B}^{N+1}$ be a smooth $C R$-embedding of a strictly pseudoconvex $C R$ real hypersurface $M^{\prime} \subset \mathbb{C}^{n+1}$. Denote $M:=H\left(M^{\prime}\right)$. If its $C R$ second fundamental 
form $I I_{M} \equiv 0$, then $M \subset F\left(\partial \mathbb{B}^{n+1}\right) \subset \partial \mathbb{B}^{N+1}$ where $F: \mathbb{B}^{n+1} \rightarrow \mathbb{B}^{N+1}$ is a certain linear fractional proper holomorphic map.

Previously, it was proved by P. Ebenfelt, X. Huang and D. Zaitsev ([EHZ04], corollary 5.5), under the above same hypothese, that $M^{\prime}$ and hence $M$ are locally CR-equivalent to the unit sphere $\partial \mathbb{B}^{n+1}$ in $\mathbb{C}^{n+1}$.

There are several definitions of the CR second fundamental forms $I I_{M}$ of $M$ (see Section 3, 4, 5, and 6). The result in [EHZ04] used Definition 1 or 2. However, to prove Theorem 1.1, we need to use Definitions 3 and 4 . We'll prove in Section 4 that $I I_{M} \equiv 0$ by any one of the four definitions will imply $I I_{M} \equiv 0$ for all other three definitions. One of the ingredients for our proof of Theorem 1.1 is the result of Ebenfelt-Huang-Zaitsev [EHZ04] so that $M$ can be regarded as the image of a rational CR map $F: \partial \mathbb{H}^{n+1} \rightarrow M \subset \partial \mathbb{H}^{N+1}$. Another ingredient is a theorem of Huang ([Hu99]) that such a map $F$ is linear if and only if its geometric rank $\kappa_{0}$ is zero. The third one is a result from [HJY09] about a special lift for maps between spheres.

Acknowledgments We would like to thank Professor Xiaojun Huang for the constant encouragement and support. The second author is also grateful to Wanke Yin and Yuan Zhang for helpful discussions.

\section{Preliminaries}

- Maps between balls We denote by $\operatorname{Prop}\left(\mathbb{B}^{n}, \mathbb{B}^{N}\right)$ the space of all proper holomorphic maps from the unit ball $\mathbb{B}^{n} \subset \mathbb{C}^{n}$ to $\mathbb{B}^{N}$, denote by $\operatorname{Prop}_{k}\left(\mathbb{B}^{n}, \mathbb{B}^{N}\right)$ the space Prop $\left(\mathbb{B}^{n}, \mathbb{B}^{N}\right) \cap$ $C^{k}\left(\overline{\mathbb{B}^{n}}\right)$, and denote by $\operatorname{Rat}\left(\mathbb{B}^{n}, \mathbb{B}^{N}\right)$ the space Prop $\left(\mathbb{B}^{n}, \mathbb{B}^{N}\right) \cap\{$ rational maps $\}$. We say that $F$ and $G \in \operatorname{Prop}\left(\mathbb{B}^{n}, \mathbb{B}^{N}\right)$ are equivalent if there are automorphisms $\sigma \in A u t\left(\mathbb{B}^{n}\right)$ and $\tau \in \operatorname{Aut}\left(\mathbb{B}^{N}\right)$ such that $F=\tau \circ G \circ \sigma$.

Write $\mathbb{H}^{n}:=\left\{(z, w) \in \mathbb{C}^{n-1} \times \mathbb{C}: \operatorname{Im}(w)>|z|^{2}\right\}$ for the Siegel upper-half space. Similarly, we can define the space $\operatorname{Prop}\left(\mathbb{H}^{n}, \mathbb{H}^{N}\right), \operatorname{Prop}_{k}\left(\mathbb{H}^{n}, \mathbb{H}^{N}\right)$ and $\operatorname{Rat}\left(\mathbb{H}^{n}, \mathbb{H}^{N}\right)$ similarly. By the Cayley transformation $\rho_{n}: \mathbb{H}^{n} \rightarrow \mathbb{B}^{n}, \rho_{n}(z, w)=\left(\frac{2 z}{1-i w}, \frac{1+i w}{1-i w}\right)$, we can identify a map $F \in \operatorname{Prop}_{k}\left(\mathbb{B}^{n}, \mathbb{B}^{N}\right)$ or $\operatorname{Rat}\left(\mathbb{B}^{n}, \mathbb{B}^{N}\right)$ with $\rho_{N}^{-1} \circ F \circ \rho_{n}$ in the space $\operatorname{Prop}_{k}\left(\mathbb{H}^{n}, \mathbb{H}^{N}\right)$ or $\operatorname{Rat}\left(\mathbb{H}^{n}, \mathbb{H}^{N}\right)$, respectively. We say that $F$ and $G \in \operatorname{Prop}\left(\mathbb{H}^{n}, \mathbb{H}^{N}\right)$ are equivalent if there are automorphisms $\sigma \in A u t\left(\mathbb{H}^{n}\right)$ and $\tau \in A u t\left(\mathbb{H}^{N}\right)$ such that $F=\tau \circ G \circ \sigma$.

We denote by $\partial \mathbb{H}^{n}=\left\{(z, w) \in \mathbb{C}^{n-1} \times \mathbb{C}: \operatorname{Im}(w)=|z|^{2}\right\}$ for the Heisenberg hypersurface. For any map $F \in \operatorname{Prop}_{2}\left(\mathbb{H}^{n}, \mathbb{H}^{N}\right)$, by restricting on $\partial \mathbb{H}^{n}$, we can regard $F$ as a $C^{2} \mathrm{CR}$ map from $\partial \mathbb{H}^{n}$ to $\partial \mathbb{H}^{N}$, and we denote it as $F \in \operatorname{Prop}_{2}\left(\partial \mathbb{H}^{n}, \partial \mathbb{H}^{N}\right)$. We say that $F$ and $G \in \operatorname{Prop}_{2}\left(\partial \mathbb{H}^{n}, \partial \mathbb{H}^{N}\right)$ are equivalent if there are automorphisms $\sigma \in \operatorname{Aut}\left(\partial \mathbb{H}^{n}\right)=\operatorname{Aut}\left(\mathbb{H}^{n}\right)$ and $\tau \in \operatorname{Aut}\left(\partial \mathbb{H}^{N}\right)=\operatorname{Aut}\left(\mathbb{H}^{N}\right)$ such that $F=\tau \circ G \circ \sigma$. 
We can parametrize $\partial \mathbb{H}^{n}$ by $(z, \bar{z}, u)$ through the map $(z, \bar{z}, u) \rightarrow\left(z, u+i|z|^{2}\right)$. In what follows, we will assign the weight of $z$ and $u$ to be 1 and 2, respectively. For a non-negative integer $m$, a function $h(z, \bar{z}, u)$ defined over a small ball $U$ of 0 in $\partial \mathbb{H}^{n}$ is said to be of quantity $o_{w t}(m)$ if $\frac{h\left(t z, t \bar{z}, t^{2} u\right)}{|t|^{m}} \rightarrow 0$ uniformly for $(z, u)$ on any compact subset of $U$ as $t(\in \mathbb{R}) \rightarrow 0$.

- Partial normalization of $F$ Let $F=(f, \phi, g)=(\widetilde{f}, g)=\left(f_{1}, \cdots, f_{n-1}, \phi_{1}, \cdots, \phi_{N-n}, g\right)$ be a non-constant map in $\operatorname{Prop}_{2}\left(\partial \mathbb{H}^{n}, \partial \mathbb{H}^{N}\right)$ with $F(0)=0$. For each $p \in \partial \mathbb{H}^{n}$, we write $\sigma_{p}^{0} \in \operatorname{Aut}\left(\mathbb{H}^{n}\right)$ with $\sigma_{p}^{0}(0)=p$ and $\tau_{p}^{F} \in \operatorname{Aut}\left(\mathbb{H}^{N}\right)$ with $\tau_{p}^{F}(F(p))=0$ for the maps

$$
\begin{aligned}
& \sigma_{p}^{0}(z, w)=\left(z+z_{0}, w+w_{0}+2 i\left\langle z, \overline{z_{0}}\right\rangle\right) \\
& \tau_{p}^{F}\left(z^{*}, w^{*}\right)=\left(z^{*}-\tilde{f}\left(z_{0}, w_{0}\right), w^{*}-\overline{g\left(z_{0}, w_{0}\right)}-2 i\left\langle z^{*}, \overline{\widetilde{f}\left(z_{0}, w_{0}\right)}\right\rangle\right) .
\end{aligned}
$$

$F$ is equivalent to $F_{p}=\tau_{p}^{F} \circ F \circ \sigma_{p}^{0}=\left(f_{p}, \phi_{p}, g_{p}\right)$. Notice that $F_{0}=F$ and $F_{p}(0)=0$. The following is basic for the understanding of the geometric properties of $F$.

Lemma 2.1 ([§2, Lemma 5.3, Hu99], [Lemma 2.0, Hu03]): Let F be a non-constant map in $\operatorname{Prop}_{2}\left(\partial \mathbb{H}^{n}, \partial \mathbb{H}^{N}\right), 2 \leq n \leq N$ with $F(0)=0$. For each $p \in \partial \mathbb{H}^{n}$, there is an automorphism $\tau_{p}^{* *} \in$ Aut $_{0}\left(\mathbb{H}^{N}\right)$ such that $F_{p}^{* *}:=\tau_{p}^{* *} \circ F_{p}$ satisfies the following normalization:

$$
\begin{gathered}
f_{p}^{* *}=z+\frac{i}{2} a_{p}^{* *(1)}(z) w+o_{w t}(3), \phi_{p}^{* *}=\phi_{p}^{* *(2)}(z)+o_{w t}(2), g_{p}^{* *}=w+o_{w t}(4), \\
\left\langle\bar{z}, a_{p}^{* *(1)}(z)\right\rangle|z|^{2}=\left|\phi_{p}^{* *(2)}(z)\right|^{2}
\end{gathered}
$$

Let $\mathcal{A}(p)=-2 i\left(\left.\frac{\partial^{2}\left(f_{p}\right)_{l}^{* *}}{\partial z_{j} \partial w}\right|_{0}\right)_{1 \leq j, l \leq n-1}$. We call the rank of $\mathcal{A}(p)$, which we denote by $R k_{F}(p)$, the geometric rank of $F$ at $p . R k_{F}(p)$ depends only on $p$ and $F$, and is a lower semi-continuous function on $p$. We define the geometric rank of $F$ to be $\kappa_{0}(F)=\max _{p \in \partial \mathbb{H}^{n}} R k_{F}(p)$. Notice that we always have $0 \leq \kappa_{0} \leq n-1$. We define the geometric rank of $F \in \operatorname{Prop}_{2}\left(\mathbb{B}^{n}, \mathbb{B}^{N}\right)$ to be the one for the map $\rho_{N}^{-1} \circ F \circ \rho_{n} \in \operatorname{Prop}_{2}\left(\mathbb{H}^{n}, \mathbb{H}^{N}\right)$.

Lemma 2.2 (ct. [Hu99], theorem 4.3) $F \in$ Prop $_{2}\left(\mathbb{B}^{n}, \mathbb{B}^{N}\right)$ has geometric rank 0 if and only if $F$ is equivalent to a linear map.

Denote by $\mathcal{S}_{0}=\left\{(j, l): 1 \leq j \leq \kappa_{0}, 1 \leq l \leq(n-1), j \leq l\right\}$ and write $\mathcal{S}:=\{(j, l):$ $(j, l) \in \mathcal{S}_{0}$, or $\left.j=\kappa_{0}+1, l \in\left\{\kappa_{0}+1, \cdots, \kappa_{0}+N-n-\frac{\left(2 n-\kappa_{0}-1\right) \kappa_{0}}{2}\right\}\right\}$. 
Lemma 2.3 ([Lemma 3.2, Hu03]): Let $F$ be a $C^{2}$-smooth $C R$ map from an open piece $M \subset \partial \mathbb{H}^{n}$ into $\partial \mathbb{H}^{N}$ with $F(0)=0$ and $R k_{F}(0)=\kappa_{0}$. Let $P\left(n, \kappa_{0}\right)=\frac{\kappa_{0}\left(2 n-\kappa_{0}-1\right)}{2}$. Then $N \geq n+P\left(n, \kappa_{0}\right)$ and there are $\sigma \in A u t_{0}\left(\partial \mathbb{H}^{n}\right)$ and $\tau \in A u t_{0}\left(\partial \mathbb{H}^{N}\right)$ such that $F_{p}^{* * *}=$ $\tau \circ F \circ \sigma:=(f, \phi, g)$ satisfies the following normalization conditions:

$$
\left\{\begin{aligned}
f_{j}= & z_{j}+\frac{i \mu_{j}}{2} z_{j} w+o_{w t}(3), \quad \frac{\partial^{2} f_{j}}{\partial w^{2}}(0)=0, j=1 \cdots, \kappa_{0}, \mu_{j}>0, \\
f_{j}= & z_{j}+o_{w t}(3), \quad j=\kappa_{0}+1, \cdots, n-1 \\
g= & w+o_{w t}(4), \\
\phi_{j l}= & \mu_{j l} z_{j} z_{l}+o_{w t}(2), \text { where }(j, l) \in \mathcal{S} \text { with } \mu_{j l}>0 \text { for }(j, l) \in \mathcal{S}_{0} \\
& \text { and } \mu_{j l}=0 \text { otherwise }
\end{aligned}\right.
$$

where $\mu_{j l}=\sqrt{\mu_{j}+\mu_{l}}$ for $j, l \leq \kappa_{0} j \neq l, \mu_{j l}=\sqrt{\mu_{j}}$ if $j \leq \kappa_{0}$ and $l>\kappa_{0}$ or if $j=l \leq \kappa_{0}$.

- Pseudohermitian metric and Webster connection Let $M$ be a $C^{2}$ smooth real hypersurface in $\mathbb{C}^{n+1}$. We denote by $T^{c} M=T M \cap i T M \subset T M$ its maximal complex tangent bundle with the complex structure $J: T^{c} M \rightarrow T^{c} M$. Here $J\left(\frac{\partial}{\partial x_{j}}\right)=\frac{\partial}{\partial y_{j}}$ and $J\left(\frac{\partial}{\partial y_{j}}\right)=-\frac{\partial}{\partial x_{j}}$ in terms of holomorphic coordinates. We denote by $\mathcal{V}=T^{0,1} M=\left\{X+i J X \mid X \in T^{c} M\right\} \subset$ $\mathbb{C} T M:=T M \otimes \mathbb{C}$ the $C R$ bundle. We also denote $T^{1,0} M=\overline{\mathcal{V}}$. All $T^{c} M, \mathcal{V}$ and $\overline{\mathcal{V}}$ are complex rank $n$ vector bundles.

Write $T^{0} M:=\left(T^{1,0} M \oplus T^{0,1} M\right)^{\perp} \subset \mathbb{C} T^{*} M$ for its rank one subbundle. Write $T^{\prime} M:=$ $T^{0,1 \perp} \subset \mathbb{C} T^{*} M$ for its rank $n+1$ holomorphic or (1,0) cotangent bundle of $M$. Here $T^{0} \subset T^{\prime} M$.

A real nonvanishing 1-form $\theta$ over $M$ is called a contact form if $\theta \wedge(d \theta)^{n} \neq 0$. Let $M$ be as above given by a defining function $r$. Then the 1 -form $\theta=i \partial r$ is a contact form of $M$.

We say that $(M, \theta)$ is strictly pseudoconvex if the Levi-form $L_{\theta}$ is positive definite for all $z \in M$. Here the Levi-form $L_{\theta}$ with respect to $\theta$ is defined by

$$
L_{\theta}(\vec{u}, \overline{\vec{v}}):=-i d \theta(\vec{u} \wedge \overline{\vec{v}}), \quad \forall \vec{u}, \vec{v} \in T_{p}^{1,0}(M), \forall p \in M .
$$

Associated with a contact form $\theta$ one has the Reeb vector field $R_{\theta}$, defined by the equations: (i) $d \theta\left(R_{\theta}, \cdot\right) \equiv 0$, (ii) $\theta\left(R_{\theta}\right) \equiv 1$. As a skew-symmetric form of maximal rank $2 n$, the form $\left.d \theta\right|_{T_{p} M}$ has a 1- dimensional kernel for each $p \in M^{2 n+1}$. Hence equation (i) defines a unique line field $\left\langle R_{\theta}\right\rangle$ on $M$. The contact condition $\theta \wedge(d \theta)^{n} \neq 0$ implies that $\theta$ is non-trivial on that line field, so the unique real vector field is defined by the normalization condition (ii). 
According Tanaka [T75] and Webester [We78, $(M, \theta)$ is called a strictly pseudoconvex pseudohermitian manifold if there are $n$ complex 1 -forms $\theta^{\alpha}$ so that $\left\{\theta^{1}, \ldots, \theta^{n}\right\}$ forms a local basis for holomorphic cotangent bundle $H^{*}(M)$ and

$$
d \theta=i \sum_{\alpha, \beta=1}^{n} h_{\alpha \bar{\beta}} \theta^{\alpha} \wedge \theta^{\bar{\beta}}
$$

where $\left(h_{\alpha \bar{\beta}}\right)$, called the Levi form matrix, is positive definite. Such $\theta^{\alpha}$ may not be unique. Following Webster (1978), a coframe $\left(\theta, \theta^{\alpha}\right)$ is called admissible if (15) holds. The admissible coframes are determined up to transformations $\widetilde{\theta}^{\alpha}=u_{\beta}^{\alpha} \theta^{\beta}$ where $\left(u_{\beta}^{\alpha}\right) \in G L\left(\mathbb{C}^{n}\right)$.

Theorem 2.4 (Webster, 1978) Let $\left(M^{2 n+1}, \theta\right)$ be a strictly pseudoconvex pseudohermitian manifold and let $\theta^{j}$ be as in (5). Then there are unique way to write

$$
d \theta^{\alpha}=\sum_{\gamma=1}^{n} \theta^{\gamma} \wedge \omega_{\gamma}^{\alpha}+\theta \wedge \tau^{\alpha},
$$

where $\tau^{\alpha}$ are $(0,1)$-forms over $M$ that are linear combination of $\theta^{\bar{\alpha}}=\overline{\theta^{\alpha}}$, and $\omega_{\alpha}^{\beta}$ are 1 -forms over $M$ such that

$$
0=d h_{\alpha \bar{\beta}}-h_{\gamma \bar{\beta}} \omega_{\alpha}^{\gamma}-h_{\alpha \bar{\gamma}} \omega_{\bar{\beta}}^{\bar{\gamma}} .
$$

We may denote $\omega_{\alpha \bar{\beta}}=h_{\gamma \bar{\beta}} \omega_{\alpha}^{\gamma}$ and $\overline{\omega_{\beta \bar{\alpha}}}=h_{\alpha \bar{\gamma}} \omega_{\bar{\beta}}^{\bar{\gamma}}$. In particular, if

$$
h_{\alpha \beta}=\delta_{\alpha \beta},
$$

the identity in (7) becomes $0=-\omega_{\alpha \bar{\beta}}-\overline{\omega_{\beta \bar{\alpha}}}$, i.e.,

$$
0=\omega_{\alpha}^{\beta}+\omega_{\bar{\beta}}^{\bar{\alpha}}
$$

The condition on $\tau^{\beta}$ means:

$$
\tau^{\beta}=A_{\bar{\nu}}^{\beta} \theta^{\bar{\nu}}, \quad A^{\alpha \beta}=A^{\beta \alpha},
$$

which holds automatically. The curvature is given by

$$
d \omega_{\alpha}^{\beta}-\omega_{\alpha}^{\gamma} \wedge \omega_{\gamma}^{\beta}=R_{\alpha}^{\beta}{ }_{\mu \nu} \theta^{\mu} \wedge \theta^{\bar{\nu}}+W_{\alpha}^{\beta}{ }_{\mu} \theta^{\mu} \wedge \theta-W_{\alpha \bar{\nu}}^{\beta} \theta^{\bar{\nu}} \wedge \theta+i \theta_{\alpha} \wedge \tau^{\beta}-i \tau_{\alpha} \wedge \theta^{\beta}
$$

where the functions $R_{\alpha}^{\beta}{ }_{\mu \bar{\nu}}$ and $W_{\alpha}{ }^{\beta}{ }_{\mu}$ represent the pseudohermitian curvature of $(M, \theta)$. 


\section{CR second fundamental forms — Definition 1}

We are going to survey four definitions of the $\mathrm{CR}$ second fundamental forms $I I_{M}$ of $M$ in $\partial \mathbb{H}^{N+1}$. We start with Definition 1 which is the intrinsic one in terms of a coframe.

Lemma 3.1 (EHZ04], corollary 4.2) Let $M$ and $\widetilde{M}$ be strictly pseudoconvex CR-manifolds of dimensions $2 n+1$ and $2 \widetilde{n}+1$ respectively, and of $C R$ dimensions $n$ and $\tilde{n}$ respectively. Let $F: M \rightarrow \widetilde{M}$ be a smooth $C R$-embedding. If $\left(\theta, \theta^{\alpha}\right)$ is a admissible coframe on $M$, then in a neighborhood of a point $\widetilde{p} \in F(M)$ in $\widetilde{M}$ there exists an admissible coframe $\left(\widetilde{\theta}, \widetilde{\theta}^{A}\right)=$ $\left(\widetilde{\theta}, \widetilde{\theta}^{\alpha}, \widetilde{\theta}^{\mu}\right)$ on $\widetilde{M}$ with $F^{*}\left(\widetilde{\theta}, \widetilde{\theta}^{\alpha}, \widetilde{\theta}^{\mu}\right)=\left(\theta, \theta^{\alpha}, 0\right)$. In particular, the Reeb vector field $\widetilde{R}$ is tangent to $F(M)$. If we choose the Levi form matrix of $M$ such that the functions $h_{\alpha \bar{\beta}}$ in (5) with respect to $\left(\theta, \theta^{\alpha}\right)$ to be $\left(\delta_{\alpha \bar{\beta}}\right)$, then $\left(\widetilde{\theta}, \widetilde{\theta}^{A}\right)$ can be chosen such that the Levi form matrix of $\widetilde{M}$ relative to it is also $\left(\delta_{A \bar{B}}\right)$. With this additional property, the coframe $\left(\widetilde{\theta}, \widetilde{\theta}^{A}\right)$ is uniquely determined along $M$ up to unitary transformations in $U(n) \times U(\widetilde{n}-n)$.

If $\left(\theta, \theta^{\alpha}\right)$ and $\left(\widetilde{\theta}, \widetilde{\theta}^{A}\right)$ are as above such that the condition on the Levi form matrices in Lemma 3.1 are satisfied, we say that the coframe $\left(\widetilde{\theta}, \widetilde{\theta}^{A}\right)$ is adapted to the coframe $\left(\theta, \theta^{\alpha}\right)$. In this case, by (9), we have $\theta=F^{*} \widetilde{\theta}, \theta^{\alpha}=F^{*} \widetilde{\theta}^{\alpha}$, and

$$
d \theta^{\alpha}=\sum_{\gamma=1}^{n} \theta^{\gamma} \wedge \omega_{\gamma}^{\alpha}+\theta \wedge \tau^{\alpha}, \quad 0=\omega_{\alpha}^{\beta}+\omega_{\bar{\beta}}^{\bar{\alpha}}, \quad \forall 1 \leq \alpha, \beta \leq n,
$$

and

$$
d \widetilde{\theta}^{A}=\sum_{B=1}^{\widetilde{n}} \widetilde{\theta}^{C} \wedge \widetilde{\omega}_{C}^{A}+\widetilde{\theta} \wedge \widetilde{\tau}^{A}, \quad 0=\widetilde{\omega}_{A}^{B}+\widetilde{\omega}_{\bar{B}}^{\bar{A}}, \quad \forall 1 \leq A, B \leq N .
$$

For simplicity, we may denote $F^{*} \widetilde{\omega}_{B}^{A}$ by $\omega_{B}^{A}$. We also denote $F^{*} \widetilde{\omega}_{A \bar{B}}$ by $\omega_{A \bar{B}}$ where $\omega_{A \bar{B}}=\omega_{A}^{B}$.

Write $\omega_{\alpha}^{\mu}=\omega_{\alpha \beta}^{\mu} \theta^{\beta}$. The matrix of $\left(\omega_{\alpha}^{\mu}{ }_{\beta}\right), 1 \leq \alpha, \beta \leq n, n+1 \leq \mu \leq \hat{n}$, defines the $C R$ second fundamental form of $M$. It was used in [We79] and [Fa90].

\section{CR second fundamental forms — Definition 2}

Definition 2 introduced in EHZ04 will be the extrinsic one in terms of defining function.

Let $F: M \rightarrow \widetilde{M}$ be a smooth CR-embedding between $M \subset \mathbb{C}^{n+1}$ and $\widetilde{M} \subset \mathbb{C}^{N+1}$ where $M$ and $\widetilde{M}$ are real strictly pseudoconvex hypersurfaces of dimensions $2 n+1$ and $2 \widetilde{n}+1$, and 
CR dimensions $n$ and $\widetilde{n}$, respectively. Let $p \in M$ and $\widetilde{p}=F(p) \in \widetilde{M}$ be points. Let $\widetilde{\rho}$ be a local defining function for $\widetilde{M}$ near the point $\widetilde{p}$. Let

$$
E_{k}(p):=\operatorname{span}_{\mathbb{C}}\left\{L^{\bar{J}}\left(\widetilde{\rho}_{Z^{\prime}} \circ F\right)(p)\left|J \in\left(Z_{+}\right)^{n}, 0 \leq\right| J \mid \leq k\right\} \subset T_{\widetilde{p}}^{1,0} \mathbb{C}^{N+1},
$$

where $\widetilde{\rho}_{Z^{\prime}}:=\partial \widetilde{\rho}$ is the complex gradient (i.e., represented by vectors in $\mathbb{C}^{N+1}$ in some local coordinate system $Z^{\prime}$ near $\widetilde{p}$ ). Here we use multi-index notation $L^{\bar{J}}=L_{1}^{\overline{J_{1}}} \cdots L_{n}^{\overline{J_{n}}}$ and $|J|=J_{1}+\ldots+J_{n}$. It was shown in [La01] that $E_{k}(p)$ is independent of the choice of local defining function $\widetilde{\rho}$, coordinates $Z^{\prime}$ and the choice of basis of the CR vector fields $L_{\overline{1}}, \ldots, L_{\bar{n}}$.

The $C R$ second fundamental form $I I_{M}$ of $M$ is defined by (cf. [EHZ04], §2)

$$
I I_{M}\left(X_{p}, Y_{p}\right):=\overline{\pi\left(X Y\left(\widetilde{\rho}_{\bar{Z}^{\prime}} \circ f\right)(p)\right)} \in \overline{T_{\tilde{p}}^{\prime} \widetilde{M} / E_{1}(p)}
$$

where $\widetilde{\rho}_{\bar{Z}^{\prime}}=\bar{\partial} \widetilde{\rho}$ is represented by vectors in $\mathbb{C}^{N+1}$ in some local coordinate system $Z^{\prime}$ near $\widetilde{p}, X, Y$ are any $(1,0)$ vector fields on $M$ extending given vectors $X_{p}, Y_{p} \in T_{p}^{1,0}(M)$, and $\pi: T_{\widetilde{p}}^{\prime} \widetilde{M} \rightarrow T_{\widetilde{p}}^{\prime} \widetilde{M} / E_{1}(p)$ is the projection map.

Since $\widetilde{M}$ and $M$ are strictly pseudoconvex, the Levi form of $\widetilde{M}$ (at $\widetilde{p}$ ) with respect to $\widetilde{\rho}$ defines an isomorphism

$$
\overline{T_{\widetilde{p}}^{\prime} \widetilde{M} / E_{1}(p)} \cong T_{\widetilde{p}}^{1,0} \widetilde{M} / F_{*}\left(T_{p}^{1,0} M\right)
$$

and the $\mathrm{CR}$ second fundamental form can be viewed as an $\mathbb{C}$-linear symmetric form

$$
I I_{M, p}: T_{p}^{1,0} M \times T_{p}^{1,0} M \rightarrow T_{\widetilde{p}}^{1,0} \widetilde{M} / F_{*}\left(T_{p}^{1,0} M\right)
$$

that does not depend on the choice of $\widetilde{\rho}$ (cf.[EHZ04], §2).

The relation between Definition 1 and Definition 2 was discussed in [EHZ04]. Let $(M, \widetilde{M})$, $\left(\theta, \theta^{\alpha}\right),\left(\widetilde{\theta}, \widetilde{\theta}^{A}\right)$ be as in Lemma 3.1, and we abuse the structure bundle $\left(\theta, \theta^{\alpha}\right)$ on $M$ with the structure bundle $\left(\widetilde{\theta}, \widetilde{\theta}^{\alpha}\right)$ on $\widetilde{M}$. We can choose a defining function $\widetilde{\rho}$ of $\widetilde{M}$ near a point $\widetilde{p}=F(p) \in \widetilde{M}$ where $p \in M$ such that $\theta=i \bar{\partial} \widetilde{\rho}$ on $\widetilde{M}$., i.e., in local coordinates $Z^{\prime}$ in $\mathbb{C}^{N+1}$, we have

$$
\theta=i \sum_{k=1}^{N+1} \frac{\partial \widetilde{\rho}}{\partial \overline{Z_{k}^{\prime}}} d \overline{Z_{k}^{\prime}},
$$

where we pull back the forms $d \overline{Z_{1}^{\prime}}, \ldots, d \overline{Z_{N+1}^{\prime}}$ to $\widetilde{M}$. Then we consider the coframe $\left(\theta, \theta^{\alpha}\right)=$ $\left(F^{*} \widetilde{\theta}, F^{*} \widetilde{\theta}^{\alpha}\right)$ on $M$ near $p$ with $F(p)=\widetilde{p}$. We take its dual frame $\left(T, L_{A}\right)$ of $\left(\theta, \theta^{A}\right)$ and have

$$
\left.L_{\beta}\left(\widetilde{\rho}_{\bar{Z}^{\prime}} \circ F\right)=-i L_{\beta}\right\lrcorner d \theta=g_{\beta \bar{C}} \theta^{\bar{C}}=g_{\beta \bar{\gamma}} \theta^{\bar{\gamma}} .
$$


Here we used the definition of the construction, (5) and the dual relationship $\left\langle L_{\beta}, \theta^{\alpha}\right\rangle=$ $\delta_{\beta}^{\alpha}$ and also notice that $g_{\beta \bar{\gamma}}=\delta_{\beta \gamma}$. Applying $L_{\alpha}$ to both sides of (14), we obtain

$$
\left.L_{\alpha} L_{\beta}\left(\widetilde{\rho}_{\bar{Z}^{\prime}} \circ F\right)=g_{\beta \bar{\gamma}} L_{\alpha}\right\lrcorner d \theta^{\bar{\gamma}}=\omega_{\alpha \bar{\mu} \beta} \theta^{\bar{\mu}} \bmod \left(\theta, \theta^{\bar{\alpha}}\right)
$$

which implies

$$
I I_{M}\left(L_{\alpha}, L_{\beta}\right)=\omega_{\alpha \beta}^{\mu} L_{\mu}, \quad n+1 \leq \mu \leq N .
$$

This identity gives the equivalent relation of the intrinsic and extrinsic definitions of $I I_{M}$. Notice that we need a right choice of $\left(\theta, \theta^{\alpha}\right),\left(T, L_{A}\right)$ and $\widetilde{\rho}$.

By using $\left(\omega_{\alpha \beta}^{b}\right)$ and (15), as in (13), we can also define

$$
I I_{M, p}: T_{p}^{1,0} M \times T_{p}^{1,0} M \rightarrow T_{\widetilde{p}}^{1,0} \widetilde{M} / F_{*}\left(T_{p}^{1,0} M\right)
$$

which is independent of the choice of the adapted coframe $\left(\theta, \theta^{A}\right)$ in case $\widetilde{M}$ is locally $\mathrm{CR}$ embeddable in $\mathbb{C}^{N+1}$ (cf. [EHZ04], § 4).

\section{CR second fundamental forms — Definition 3}

Definition 3 will be the one as a tensor with respect to the group $G L^{Q}\left(\mathbb{C}^{N+2}\right)$.

The bundle $G L^{Q}\left(\mathbb{C}^{N+2}\right)$ over $\partial \mathbb{H}^{N+1} \quad$ We consider a real hypersurface $Q$ in $\mathbb{C}^{N+2}$ defined by the homogeneous equation

$$
\langle Z, Z\rangle:=\sum_{A} Z^{A} \overline{Z^{A}}+\frac{i}{2}\left(\overline{Z^{0}} Z^{N+1}-Z^{0} \overline{Z^{N+1}}\right)=0,
$$

where $Z=\left(Z^{0}, Z^{A}, Z^{N+1}\right)^{t} \in \mathbb{C}^{N+2}$. Let

$$
\pi_{0}: \mathbb{C}^{N+2}-\{0\} \rightarrow \mathbb{C P}^{N+1}, \quad\left(z_{0}, \ldots, z_{N+1}\right) \mapsto\left[z_{0}: \ldots: z_{N+1}\right],
$$

be the standard projection. For any point $x \in \mathbb{C P}^{N+1}, \pi_{0}^{-1}(x)$ is a complex line in $\mathbb{C}^{N+2}-\{0\}$. For any point $v \in \mathbb{C}^{N+2}-\{0\}, \pi_{0}(v) \in \mathbb{C P}^{N+1}$ is a point. The image $\pi_{0}(Q-\{0\})$ is the Heisenberg hypersurface $\partial \mathbb{H}^{N+1} \subset \mathbb{C P}^{N+1}$.

For any element $A \in G L\left(\mathbb{C}^{N+2}\right)$ :

$$
A=\left(a_{0}, \ldots, a_{N+1}\right)=\left[\begin{array}{cccc}
a_{0}^{(0)} & a_{1}^{(0)} & \ldots & a_{N+1}^{(0)} \\
a_{0}^{(1)} & a_{1}^{(1)} & \ldots & a_{N+1}^{(1)} \\
\vdots & \vdots & & \vdots \\
a_{0}^{(N+1)} & a_{1}^{(N+1)} & \ldots & a_{N+1}^{(N+1)}
\end{array}\right] \in G L\left(\mathbb{C}^{N+2}\right)
$$


where each $a_{j}$ is a column vector in $\mathbb{C}^{N+2}, 0 \leq j \leq N+1$. This $A$ is associated to an automorphism $A^{\star} \in \operatorname{Aut}\left(\mathbb{C P}^{N+1}\right)$ given by

$$
A^{\star}\left(\left[z_{0}: z_{1}: \ldots: z_{N+1}\right]\right)=\left[\sum_{j=0}^{N+1} a_{j}^{(0)} z_{j}: \sum_{j=0}^{N+1} a_{j}^{(1)} z_{j}: \ldots: \sum_{j=0}^{N+1} a_{j}^{(N+1)} z_{j}\right] .
$$

When $a_{0}^{(0)} \neq 0$, in terms of the non-homogeneous coordinates $\left(w_{1}, \ldots, w_{n}\right), A^{\star}$ is a linear fractional from $\mathbb{C}^{N+1}$ which is holomorphic near $(0, \ldots, 0)$ :

$$
A^{\star}\left(w_{1}, \ldots, w_{N+1}\right)=\left(\frac{\sum_{j=0}^{N+1} a_{j}^{(1)} w_{j}}{\sum_{j=0}^{N+1} a_{j}^{(0)} w_{j}}, \ldots, \frac{\sum_{j=0}^{N+1} a_{j}^{(N+1)} w_{j}}{\sum_{j=0}^{N+1} a_{j}^{(0)} w_{j}}\right), \quad \text { where } w_{j}=\frac{z_{j}}{z_{0}} .
$$

We denote $A \in G L^{Q}\left(\mathbb{C}^{N+2}\right)$ if $A$ satisfies $A(Q) \subseteq Q$ where we regard $A$ as a linear transformation of $\mathbb{C}^{N+2}$. If $A \in G L^{Q}\left(\mathbb{C}^{N+2}\right)$, we must have $A^{\star}\left(\partial \mathbb{H}^{N+1}\right) \subseteq \partial \mathbb{H}^{N+1}$, so that $A^{\star} \in A u t\left(\partial \mathbb{H}^{N+1}\right)$. Conversely, if $A^{\star} \in A u t\left(\partial \mathbb{H}^{N+1}\right)$, then $A \in G L^{Q}\left(\mathbb{C}^{N+2}\right)$.

We define a bundle map:

$$
\begin{aligned}
& \pi: \quad G L\left(\mathbb{C}^{N+2}\right) \quad \rightarrow \quad \mathbb{C P}^{N+1} \\
& A=\left(a_{0}, a_{1}, \ldots, a_{N+1}\right) \mapsto \pi_{0}\left(a_{0}\right) .
\end{aligned}
$$

Then by (20), for any map $A \in G L\left(\mathbb{C}^{N+2}\right), A \in \pi^{-1}\left(\pi_{0}\left(a_{0}\right)\right) \Longleftrightarrow A^{\star}([1: 0: \ldots: 0])=$ $\pi_{0}\left(a_{0}\right)$. In particular, by the restriction, we consider a map

$$
\begin{aligned}
\pi: \quad G L^{Q}\left(\mathbb{C}^{N+2}\right) & \rightarrow \partial \mathbb{H}^{N+1} \\
A=\left(a_{0}, a_{1}, \ldots, a_{N+1}\right) & \mapsto \pi_{0}\left(a_{0}\right) .
\end{aligned}
$$

We get $\partial \mathbb{H}^{N+1} \simeq G L^{Q}\left(\mathbb{C}^{N+2}\right) / P_{1}$ where $P_{1}$ is the isotropy subgroup of $G L^{Q}\left(\mathbb{C}^{N+2}\right)$. Then by (20), for any map $A \in G L^{Q}\left(\mathbb{C}^{n+2}\right)$,

$$
A \in \pi^{-1}\left(\pi_{0}\left(a_{0}\right)\right) \Longleftrightarrow A^{\star}([1: 0: \ldots: 0])=\pi_{0}\left(a_{0}\right) .
$$

CR submanifolds of $\partial \mathbb{H}^{N+1} \quad$ Let $H: M^{\prime} \rightarrow \partial \mathbb{H}^{N+1}$ be a CR smooth embedding where $M^{\prime}$ is a strictly pseudoconvex smooth real hypersurface in $\mathbb{C}^{n+1}$. We denote $M=H\left(M^{\prime}\right)$.

Let $R_{M^{\prime}}$ be the Reeb vector field of $M^{\prime}$ with respect to a fixed contact form on $M^{\prime}$. Then the real vector $R_{M^{\prime}}$ generates a real line bundle over $M^{\prime}$, denoted by $\mathcal{R}_{M^{\prime}}$. Since we can regard the rank $n$ complex vector bundle $T^{1,0} M^{\prime}$ as the rank $2 n$ real vector bundle, over the real number field $\mathbb{R}$ we have:

$$
T M^{\prime}=T^{c} M^{\prime} \oplus \mathcal{R}_{M^{\prime}} \simeq T^{1,0} M^{\prime} \oplus \mathcal{R}_{M^{\prime}}
$$


given by

$$
\left(a_{j} \frac{\partial}{\partial x_{j}}, b_{j} \frac{\partial}{\partial y_{j}}\right)+c R_{M^{\prime}} \mapsto\left(a_{j}+i b_{j}\right) \frac{\partial}{\partial z_{j}}+c R_{M^{\prime}}, \quad \forall a_{j}, b_{j}, c \in \mathbb{R} .
$$

Since $H$ is a CR embedding, we have

$$
H_{*}\left(T^{1,0} M^{\prime}\right)=T^{1,0} M \subset T^{1,0}\left(\partial \mathbb{H}^{N+1}\right), T M \simeq H_{*}\left(T^{1,0} M^{\prime}\right) \oplus H_{*}\left(\mathcal{R}_{M^{\prime}}\right) \subset T\left(\partial \mathbb{H}^{N+1}\right) .
$$

Lifts of the CR submanifolds Let $M=H\left(M^{\prime}\right) \subset \partial \mathbb{H}^{N+1}$ be as above. Consider the commutative diagram

$$
\begin{array}{ccc} 
& & G L^{Q}\left(\mathbb{C}^{N+2}\right) \\
& e \nearrow & \downarrow \\
\hookrightarrow & \hookrightarrow & \partial \mathbb{H}^{N+1}
\end{array}
$$

Any map $e$ satisfying $\pi \circ e=I d$ is called a lift of $M$ to $G L^{Q}\left(\mathbb{C}^{N+2}\right)$.

In order to define a more specific lifts, we need to give some relationship between geometry on $\partial \mathbb{H}^{N+1}$ and on $\mathbb{C}^{N+2}$ as follows. For any subset $X \in \partial \mathbb{H}^{N+1}$, we denote $\hat{X}:=\pi_{0}^{-1}(X)$ where $\pi_{0}: \mathbb{C}^{N+2}-\{0\} \rightarrow \mathbb{C P}^{N+1}$ is the standard projection map (18). In particular, for any $x \in M, \hat{x}$ is a complex line and for the real submanifold $M^{2 n+1}$, the real submanifold $\hat{M}^{2 n+3}$ is of dimension $2 n+3$.

For any $x \in M$, we take $v \in \hat{x}=\pi_{0}^{-1}(x) \subset \mathbb{C}^{N+2}-\{0\}$, and we define

$$
\hat{T}_{x} M=T_{v} \hat{M}, \quad \hat{T}_{x}^{1,0} M=T_{v}^{1,0} \hat{M}, \quad \hat{\mathcal{R}}_{M, x}:=\mathcal{R}_{\hat{M}, v}
$$

where $\mathcal{R}_{\hat{M}}=\cup_{v \in \hat{M}} \mathcal{R}_{\hat{M}, v}$. These definitions are independent of choice of $v$.

A lift $e=\left(e_{0}, e_{\alpha}, e_{\mu}, e_{N+1}\right)$ of $M$ into $G L^{Q}\left(\mathbb{C}^{N+2}\right)$, where $1 \leq \alpha \leq n$ and $n+1 \leq \mu \leq N$, is called a first-order adapted lift if it satisfies the conditions:

$$
e_{0}(x) \in \pi_{0}^{-1}(x), \operatorname{span}_{\mathbb{C}}\left(e_{0}, e_{\alpha}\right)(x)=\hat{T}_{x}^{1,0} M, \quad \operatorname{span}\left(e_{0}, e_{\alpha}, e_{N+1}\right)(x)=\hat{T}_{x}^{1,0} M \oplus \hat{\mathcal{R}}_{M, x}
$$

where

$$
\operatorname{span}\left(e_{0}, e_{\alpha}, e_{N+1}\right)(x):=\left\{c_{0} e_{0}+c_{\alpha} e_{\alpha}+c_{N+1} e_{N+1} \mid c_{0}, c_{\alpha} \in \mathbb{C}, c_{N+1} \in \mathbb{R}\right\} .
$$

Here we used (25) and the fact that the Reeb vector is real. Locally first-order adapted lifts always exist (see Theorem 7.1 below).

We have the restriction bundle $\mathcal{F}_{M}^{0}:=\left.G L^{Q}\left(\mathbb{C}^{N+2}\right)\right|_{M}$ over $M$. The subbundle $\pi: \mathcal{F}_{M}^{1} \rightarrow$ $M$ of $\mathcal{F}_{M}^{0}$ is defined by

$$
\mathcal{F}_{M}^{1}=\left\{\left(e_{0}, e_{j}, e_{\mu}, e_{N+1}\right) \in \mathcal{F}_{M}^{0} \mid\left[e_{0}\right] \in M, \text { (27) are satisfied }\right\} .
$$


Local sections of $\mathcal{F}_{M}^{1}$ are exactly all local first-order adapted lifts of $M$.

For two first-order adapted lifts $s=\left(e_{0}, e_{j}, e_{\mu}, e_{N+1}\right)$ and $\widetilde{s}=\left(\widetilde{e}_{0}, \widetilde{e}_{j}, \widetilde{e}_{\mu}, \widetilde{e}_{N+1}\right)$, by (27), we have

$$
\left\{\begin{array}{l}
\widetilde{e}_{0}=g_{0}^{0} e_{0} \\
\widetilde{e}_{j}=g_{j}^{0} e_{0}+g_{j}^{k} e_{k} \\
\widetilde{e}_{\mu}=g_{\mu}^{0} e_{0}+g_{\mu}^{j} e_{j}+g_{\mu}^{\nu} e_{\nu}+g_{\mu}^{N+1} e_{N+1} \\
\widetilde{e}_{N+1}=g_{N+1}^{0} e_{0}+g_{N+1}^{j} e_{j}+g_{N+1}^{N+1} e_{N+1}
\end{array}\right.
$$

Notice that by (25), $g_{N+1}^{N+1}$ is some real-valued function, while other are complex-valued functions. In other words, $\widetilde{s}=s \cdot g$ where

$$
g=\left(g_{0}, g_{j}, g_{\mu}, g_{N+1}\right)=\left(\begin{array}{cccc}
g_{0}^{0} & g_{k}^{0} & g_{\mu}^{0} & g_{N+1}^{0} \\
0 & g_{k}^{j} & g_{\mu}^{j} & g_{N+1}^{j} \\
0 & 0 & g_{\mu}^{\nu} & 0 \\
0 & 0 & g_{\mu}^{N+1} & g_{N+1}^{N+1}
\end{array}\right)
$$

is a smooth map from $M$ into $G L^{Q}\left(\mathbb{C}^{N+2}\right)$. Then the fiber of $\pi: \mathcal{F}_{M}^{1} \rightarrow M$ over a point is isomorphic to the group

$$
G_{1}=\left\{g=\left(\begin{array}{cccc}
g_{0}^{0} & g_{\beta}^{0} & g_{\mu}^{0} & g_{N+1}^{0} \\
0 & g_{\beta}^{\alpha} & g_{\mu}^{\alpha} & g_{N+1}^{\alpha} \\
0 & 0 & g_{\mu}^{\nu} & 0 \\
0 & 0 & g_{\mu}^{N+1} & g_{N+1}^{N+1}
\end{array}\right) \in G L^{Q}\left(\mathbb{C}^{N+2}\right)\right\}
$$

where we use the index ranges $1 \leq \alpha, \beta \leq n$ and $n+1 \leq \mu, \nu \leq N$.

We pull back the Maurer-Cartan form from $G L^{Q}\left(\mathbb{C}^{N+2}\right)$ to $\mathcal{F}_{M}^{1}$ by a first-order adapted lift $e$ of $M$ as

$$
\omega=\left(\begin{array}{cccc}
\omega_{0}^{0} & \omega_{\beta}^{0} & \omega_{\nu}^{0} & \omega_{N+1}^{0} \\
\omega_{0}^{\alpha} & \omega_{\beta}^{\alpha} & \omega_{\nu}^{\alpha} & \omega_{N+1}^{\alpha} \\
\omega_{0}^{\mu} & \omega_{\beta}^{\mu} & \omega_{\nu}^{\mu} & \omega_{N+1}^{\mu} \\
\omega_{0}^{N+1} & \omega_{\beta}^{N+1} & \omega_{\nu}^{N+1} & \omega_{N+1}^{N+1}
\end{array}\right)
$$

Since $\omega=e^{-1} d e$, i.e., $e \omega=d e$. Then we have

$$
d e_{0}=e_{0} \omega_{0}^{0}+e_{\alpha} \omega_{0}^{\alpha}+e_{\mu} \omega_{0}^{\mu}+e_{N+1} \omega_{0}^{N+1} .
$$

On the other hand, we claim:

$$
d e_{0}=e_{0} \omega_{0}^{0}+e_{\alpha} \omega_{0}^{\alpha}+e_{N+1} \omega_{0}^{N+1} .
$$


In fact, take local coordinates systems $\left(x_{1}, \ldots, x_{2 n+1}\right)$ for the real manifold $M$, and $\left(y_{1}, y_{2}, x_{1}\right.$, ..., $\left.x_{2 n+1}\right)$ for the real manifold $\hat{M}$ where $\left(y_{1}, y_{2}\right)$ is the coordinates for fibers. By the first condition in (27), fixing $x_{1}, \ldots, x_{j-1}, x_{j+1}, \ldots, x_{2 n+1}, e_{0}\left(\ldots, x_{j}, \ldots\right)$ is a curve into $M$ with parameter $x_{j}$. Then $\frac{\partial e_{0}}{\partial x_{j}} \in T \hat{M}$ is a tangent vector to this curve. Since $\operatorname{span}\left(e_{0}, e_{\alpha}, e_{N+1}\right)(x)=$ $\hat{T}_{x}^{1,0} M \oplus \hat{\mathcal{R}}_{M, x}$ in (27) and $T \hat{M} \cong T^{1,0} \hat{M} \oplus \mathcal{R}_{\hat{M}}$, we obtain

$$
\frac{\partial e_{0}}{\partial x_{j}}=b_{0}^{j} e_{0}+b_{\alpha}^{j} e_{\alpha}+b_{N+1}^{j} e_{N+1}, \quad 1 \leq j \leq 2 n+1
$$

for some functions $b_{0}^{j}, b_{\alpha}^{j}$ and $b_{N+1}^{j}$. We also have

$$
\frac{\partial e_{0}}{\partial y_{i}}=0, \quad \text { for } i=1,2,
$$

because $\left(y_{1}, y_{2}\right)$ are the coordinates for fibers. From (33) and (34), we get

$$
\begin{gathered}
d e_{0}=\frac{\partial e_{0}}{\partial y_{1}} d y_{1}+\frac{\partial e_{0}}{\partial y_{2}} d y_{2}+\sum_{j} \frac{\partial e_{0}}{\partial x_{j}} d x_{j}=\sum_{j}\left(b_{0}^{j} e_{0}+b_{\alpha}^{j} e_{\alpha}+b_{N+1}^{j} e_{N+1}\right) d x_{j} \\
=\left(\sum_{j} b_{0}^{j} d x_{j}\right) e_{0}+\left(\sum_{j} b_{\alpha}^{j} d x_{j}\right) e_{\alpha}+\left(\sum_{j} b_{N+1}^{j} d x_{j}\right) e_{N+1} .
\end{gathered}
$$

Since the 1-forms $\omega_{0}^{0}, \omega_{0}^{\alpha}, \omega_{N+1}^{\alpha}$ in (31) are unique, from (35), it proves Claim (32).

By (311) and (32), we conclude $\omega_{0}^{\mu}=0, \forall \mu$. By the Maurer-Cartan equation $d \omega=-\omega \wedge \omega$, one gets $0=d \omega_{0}^{\nu}=-\omega_{\alpha}^{\nu} \wedge \omega_{0}^{\alpha}-\omega_{N+1}^{\nu} \wedge \omega_{0}^{N+1}$, i.e., $0=-\omega_{\alpha}^{\nu} \wedge \omega_{0}^{\alpha}, \bmod \left(\omega_{0}^{N+1}\right)$. Then by Cartan's lemma,

$$
\omega_{\beta}^{\nu}=q_{\alpha \beta}^{\nu} \omega_{0}^{\alpha} \bmod \left(\omega_{0}^{N+1}\right),
$$

for some functions $q_{\alpha \beta}^{\nu}=q_{\beta \alpha}^{\nu}$.

The CR second fundamental form In order to define the CR second fundamental form $I I_{M}=I I_{M}^{s}=q_{\alpha \beta}^{\mu} \omega_{0}^{\alpha} \omega_{0}^{\beta} \otimes \underline{e}_{\mu}, \bmod \left(\omega_{0}^{N+1}\right)$, let us define $\underline{e}_{\mu}$ as follows.

For any first-order adapted lift $e=\left(e_{0}, e_{\alpha}, e_{\nu}, e_{N+1}\right)$ with $\pi_{0}\left(e_{0}\right)=x$, we have $e_{\alpha} \in \hat{T}_{x}^{1,0} M$. Recall $T_{E} G(k, V) \simeq E^{*} \otimes(V / E)$ where $G(k, V)$ is the Grassmannian of $k$-planes that pass through the origin in a vector space $V$ over $\mathbb{R}$ or $\mathbb{C}$ and $E \in G(k, V)$ ([IL03], p.73). Then $T_{x} M \simeq(\hat{x})^{*} \otimes\left(\hat{T}_{x} M / \hat{x}\right)$ and hence the vector $e_{\alpha}$ induces $\underline{e_{\alpha}} \in T_{x}^{1,0} M$ by

$$
\underline{e}_{\alpha}=e^{0} \otimes\left(e_{\alpha} \bmod \left(e_{0}\right)\right),
$$

where we denote by $\left(e^{0}, e^{\alpha}, e^{\mu}, e^{N+1}\right)$ the dual basis of $\left(\mathbb{C}^{N+2}\right)^{*}$. Similarly, we let

$$
\underline{e}_{\mu}=e^{0} \otimes\left(e_{\mu} \bmod \hat{T}_{x}^{(1,0)} M\right) \in N_{x}^{1,0} M,
$$


where $N^{1,0} M$ is the CR normal bundle of $M$ defined by $N_{x}^{1,0} M=T_{x}^{1,0}\left(\partial \mathbb{H}^{N+1}\right) / T_{x}^{1,0} M$.

By direct computation, we obtain a tensor

$$
I I_{M}=I I_{M}^{e}=q_{\alpha \beta}^{\mu} \omega_{0}^{\alpha} \omega_{0}^{\beta} \otimes \underline{e}_{\mu} \in \Gamma\left(M, S^{2} T_{\pi_{0}\left(e_{0}\right)}^{1,0 *} M \otimes N_{\pi_{0}\left(e_{0}\right)}^{1,0} M\right) \bmod \left(\omega_{0}^{N+1}\right) .
$$

The tensor $I I_{M}$ is called the $C R$ second fundamental form of $M$.

Pulling back a lift Let $M \subset \partial \mathbb{H}^{N+1}$ be as above with a point $Q_{0} \in M$. Let $A \in G L^{Q}\left(\mathbb{C}^{N+2}\right), A^{\star} \in A u t\left(\partial \mathbb{H}^{N+1}\right)$ with $A^{\star}\left(Q_{0}\right)=P_{0}$ and $\widetilde{M}=A^{\star}(M)$. Let $\widetilde{s}: \widetilde{M} \rightarrow$ $G L^{Q}\left(\mathbb{C}^{N+2}\right)$ be a lift. We claim:

$$
s:=A^{-1} \cdot \widetilde{s} \circ A^{\star},
$$

is also a lift from $M$ into $G L^{Q}\left(\mathbb{C}^{N+2}\right)$. In fact, in order to prove that $s$ is a lift, it suffices to prove: $\pi s=I d$, i.e., for any point $Q \in M$ near $Q_{0}, \pi s(Q)=Q$. In fact,

$$
\pi s(Q)=\pi\left(A^{-1} \cdot \widetilde{s} \circ A^{\star}\right)(Q)=\pi\left(A^{-1} \cdot \widetilde{s}(P)\right)=\left(A^{\star}\right)^{-1}(\pi \widetilde{s}(P))=\left(A^{\star}\right)^{-1}(P)=Q .
$$

so that our claim is proved.

If, in addition, $\widetilde{s}$ is a first-order adapted lift of $\widetilde{M}$ into $G L^{Q}\left(\mathbb{C}^{N+2}\right), s$ is also a first-order adapted lift of $M$ into $G L^{Q}\left(\mathbb{C}^{N+2}\right)$.

Let $\Omega$ be the Maurer-Cartan form over $G L^{Q}\left(\mathbb{C}^{N+2}\right)$. Then by the invariant property $A^{*} \Omega=\Omega$, we have $s^{*} \Omega=\left(A^{-1} \cdot \widetilde{s} \circ A^{\star}\right)^{*} \Omega=\left(A^{\star}\right)^{*}(\widetilde{s})^{*}\left(A^{-1}\right)^{*} \Omega=\left(A^{\star}\right)^{*}(\widetilde{s})^{*} \Omega$, i.e., it holds on $M$ that

$$
\omega=\left(A^{\star}\right)^{*} \widetilde{\omega}
$$

where $\omega=s^{*} \Omega$ and $\widetilde{\omega}=\widetilde{s}^{*} \Omega$ so that $\omega_{0}^{\alpha}=\left(A^{\star}\right)^{*} \widetilde{\omega}_{0}^{\alpha}$ and $\omega_{\beta}^{\mu}=\left(A^{\star}\right)^{*} \widetilde{\omega}_{\beta}^{\mu}$. The last equality yields

$$
q_{\alpha \beta}^{\mu}=\widetilde{q}_{\alpha \beta}^{\mu} \circ A^{\star}
$$

\section{CR second fundamental forms — Definition 4}

Definition 4 will be the one as a tensor with respect to the group $S U(N+1,1)$.

As for Definition 3, we consider the real hypersurface $Q$ in $\mathbb{C}^{N+2}$ defined by the homogeneous equation

$$
\langle Z, Z\rangle:=\sum_{A} Z^{A} \overline{Z^{A}}+\frac{i}{2}\left(Z^{N+1} \overline{Z^{0}}-Z^{0} \overline{Z^{N+1}}\right)=0
$$


where $Z=\left(Z^{0}, Z^{A}, Z^{N+1}\right)^{t} \in \mathbb{C}^{N+2}$. This can be extended to the scalar product

$$
\left\langle Z, Z^{\prime}\right\rangle:=\sum_{A} Z^{A} \overline{Z^{\prime A}}+\frac{i}{2}\left(Z^{N+1}{\overline{Z^{\prime}}}^{0}-Z^{0} \overline{Z^{\prime N+1}}\right),
$$

for any $Z=\left(Z^{0}, Z^{A}, Z^{N+1}\right)^{t}, Z^{\prime}=\left(Z^{\prime 0}, Z^{\prime A}, Z^{\prime N+1}\right)^{t} \in \mathbb{C}^{N+2}$. This product has the properties: $\left\langle Z, Z^{\prime}\right\rangle$ is linear in $Z$ and anti-linear in $Z^{\prime} ; \overline{\left\langle Z, Z^{\prime}\right\rangle}=\left\langle Z^{\prime}, Z\right\rangle$; and $Q$ is defined by $\langle Z, Z\rangle=0$.

Let $S U(N+1,1)$ be the group of unimodular linear transformations of $\mathbb{C}^{N+2}$ that leave the form $\langle Z, Z\rangle$ invariant (cf. [CM74]).

By a $Q$-frame is meant an element $E=\left(E_{0}, E_{A}, E_{N+1}\right) \in G L\left(\mathbb{C}^{N+2}\right)$ satisfying (cf. $[\mathrm{CM} 74,(1.10)])$

$$
\left\{\begin{array}{l}
\operatorname{det}(E)=1 \\
\left\langle E_{A}, E_{B}\right\rangle=\delta_{A B},\left\langle E_{0}, E_{N+1}\right\rangle=-\left\langle E_{N+1}, E_{0}\right\rangle=-\frac{i}{2},
\end{array}\right.
$$

while all other products are zero.

There is exactly one transformation of $S U(N+1,1)$ which maps a given $Q$-frame into another. By fixing one $Q$-frame as reference, the group $S U(N+1,1)$ can be identified with the space of all $Q$-frames. Then $S U(N+1,1) \subset G L^{Q}\left(\mathbb{C}^{N+1}\right)$ is a subgroup with the composition operation. By (22) and the restriction, we have the projection

$$
\pi: S U(N+1,1) \rightarrow \partial \mathbb{H}^{N+1},\left(Z_{0}, Z_{A}, Z_{N+1}\right) \mapsto \operatorname{span}\left(Z_{0}\right)
$$

which is called a $Q$-frames bundle. We get $\partial \mathbb{H}^{N+1} \simeq S U(N+1,1) / P_{2}$ where $P_{2}$ is the isotropy subgroup of $S U(N+1,1)$. $S U(N+1,1)$ acts on $\partial \mathbb{H}^{N+1}$ effectively.

Consider $E=\left(E_{0}, E_{A}, E_{N+1}\right) \in S U(N+1,1)$ as a local lift. Then the Maurer-Cartan form $\Theta$ on $S U(N+1,1)$ is defined by $d E=\left(d E_{0}, d E_{A}, d E_{N+1}\right)=E \Theta$, or $\Theta=E^{-1} \cdot d E$, i.e.,

$$
d\left(\begin{array}{lll}
E_{0} & E_{A} & E_{N+1}
\end{array}\right)=\left(\begin{array}{lll}
E_{0} & E_{B} & E_{N+1}
\end{array}\right)\left(\begin{array}{ccc}
\Theta_{0}^{0} & \Theta_{A}^{0} & \Theta_{N+1}^{0} \\
\Theta_{0}^{B} & \Theta_{A}^{B} & \Theta_{N+1}^{B} \\
\Theta_{0}^{N+1} & \Theta_{A}^{N+1} & \Theta_{N+1}^{N+1}
\end{array}\right)
$$

where $\Theta_{A}^{B}$ are 1-forms on $S U(N+1,1)$. By (43) and (45), the Maurer-Cartan form $(\Theta)$ satisfies

$$
\begin{aligned}
& \Theta_{0}^{0}+\overline{\Theta_{N+1}^{N+1}}=0, \Theta_{0}^{N+1}=\overline{\Theta_{0}^{N+1}}, \Theta_{N+1}^{0}=\overline{\Theta_{N+1}^{0}}, \\
& \Theta_{A}^{N+1}=2 i \overline{\Theta_{0}^{A}}, \Theta_{N+1}^{A}=-\frac{i}{\Theta_{A}^{0}}, \Theta_{B}^{A}+\overline{\Theta_{A}^{B}}=0, \Theta_{0}^{0}+\Theta_{A}^{A}+\Theta_{N+1}^{N+1}=0,
\end{aligned}
$$


where $1 \leq A \leq N$. For example, from $\left\langle E_{A}, E_{B}\right\rangle=\delta_{A B}$, by taking differentiation, we obtain

$$
\left\langle d E_{A}, E_{B}\right\rangle+\left\langle E_{A}, d E_{B}\right\rangle=0 .
$$

By (45), we have

$$
\left\{\begin{array}{l}
d E_{0}=E_{0} \Theta_{0}^{0}+E_{B} \Theta_{0}^{B}+E_{N+1} \Theta_{0}^{N+1} \\
d E_{A}=E_{0} \Theta_{A}^{0}+E_{B} \Theta_{A}^{B}+E_{N+1} \Theta_{A}^{N+1} \\
d E_{N+1}=E_{0} \Theta_{N+1}^{0}+E_{B} \Theta_{N+1}^{B}+E_{N+1} \Theta_{N+1}^{N+1} .
\end{array}\right.
$$

Then

$$
\left\langle E_{0} \Theta_{A}^{0}+E_{C} \Theta_{A}^{C}+E_{N+1} \Theta_{A}^{N+1}, E_{B}\right\rangle+\left\langle E_{A}, E_{0} \Theta_{B}^{0}+E_{D} \Theta_{B}^{D}+E_{N+1} \Theta_{B}^{N+1}\right\rangle=0,
$$

which implies $\Theta_{A}^{B}+\overline{\Theta_{B}^{A}}=0$. In particular, from (46), $\Theta_{A}^{0}=-2 i \overline{\Theta_{N+1}^{A}} \cdot \Theta$ satisfies

$$
d \Theta=-\Theta \wedge \Theta .
$$

Let $M \hookrightarrow \partial \mathbb{H}^{N+1}$ be the image of $H: M^{\prime} \rightarrow \partial \mathbb{H}^{N+1}$ where $M^{\prime} \subset \mathbb{C}^{n+1}$ is a CR strictly pseudoconvex smooth hypersurface. Consider the inclusion map $M \hookrightarrow \partial \mathbb{H}^{N+1}$ and a lift $e=\left(e_{0}, e_{1}, \ldots, e_{N+1}\right)=\left(e_{0}, e_{\alpha}, e_{\nu}, e_{N+1}\right)$ of $M$ where $1 \leq \alpha \leq n$ and $n+1 \leq \nu \leq N$

$$
\begin{array}{ccc} 
& & S U(N+1,1) \\
M & \hookrightarrow & \partial \mathbb{H}^{N+1}
\end{array}
$$

We call $e$ a first-order adapted lift if for any $x \in M$,

$$
\pi_{0}\left(e_{0}(x)\right)=x, \operatorname{span}_{\mathbb{C}}\left(e_{0}, e_{\alpha}\right)(x)=\hat{T}_{x}^{1,0} M, \operatorname{span}\left(e_{0}, e_{\alpha}, e_{N+1}\right)(x)=\hat{T}_{x}^{1,0} M \oplus \hat{\mathcal{R}}_{M, x} .
$$

Locally first-order adapted lifts always exist (see Theorem 7.1 below). We have the restriction bundle $\mathcal{F}_{M}^{0}:=\left.S U(N+1,1)\right|_{M}$ over $M$. The subbundle $\pi: \mathcal{F}_{M}^{1} \rightarrow M$ of $\mathcal{F}_{M}^{0}$ is defined by

$$
\mathcal{F}_{M}^{1}=\left\{\left(e_{0}, e_{j}, e_{\mu}, e_{N+1}\right) \in \mathcal{F}_{M}^{0} \mid\left[e_{0}\right] \in M, \text { (48) are satisfied }\right\} .
$$

Local sections of $\mathcal{F}_{M}^{1}$ are exactly all local first-order adapted lifts of $M$. The fiber of $\pi$ : $\mathcal{F}_{M}^{1} \rightarrow M$ over a point is isomorphic to the group

$$
G_{1}=\left\{g=\left(\begin{array}{cccc}
g_{0}^{0} & g_{\beta}^{0} & g_{\nu}^{0} & g_{N+1}^{0} \\
0 & g_{\beta}^{\alpha} & g_{\nu}^{\alpha} & g_{N+1}^{\alpha} \\
0 & 0 & g_{\nu}^{\mu} & 0 \\
0 & 0 & 0 & g_{N+1}^{N+1}
\end{array}\right) \in S U(N+1,1)\right\}
$$


where we use the index ranges $1 \leq \alpha, \beta \leq n$ and $n+1 \leq \mu, \nu \leq N$.

By the remark below (29), $g_{N+1}^{N+1}$ is real-valued. By (43), we have $\left\langle g_{0}, g_{N+1}\right\rangle=-\frac{i}{2}$, it implies $g_{0}^{0} \cdot \overline{g_{N+1}^{N+1}}=1$. In particular, both $g_{N+1}^{N+1}$ and $g_{0}^{0}$ are real. Since $\left\langle g_{0}, g_{\mu}\right\rangle=0$ and $g_{0}^{0} \neq 0$, it implies $g_{\mu}^{N+1}=0$. Since $\left\langle g_{\alpha}, g_{\beta}\right\rangle=\delta_{\alpha \beta}$, it implies that the matrix $\left(g_{\alpha}^{\beta}\right)$ is unitary. Since $\operatorname{deg}(g)=1$, it implies $g_{0}^{0} \cdot \operatorname{det}\left(g_{\alpha}^{\beta}\right) \cdot \operatorname{det}\left(g_{\mu}^{\nu}\right) \cdot g_{N+1}^{N+1}=1$, i.e., $\operatorname{det}\left(g_{\alpha}^{\beta}\right) \cdot \operatorname{det}\left(g_{\mu}^{\nu}\right)=1$.

By considering all first-order adapted lifts from $M$ into $S U(N+1,1)$, as the definition of $I I_{M}$ in Definition 3, we can defined CR second fundamental form $I I_{M}$ as in (37):

$$
I I_{M}=I I_{M}^{e}=q_{\alpha \beta}^{\mu} \omega_{0}^{\alpha} \omega_{0}^{\beta} \otimes \underline{e}_{\mu} \in \Gamma\left(M, S^{2} T_{\pi_{0}\left(e_{0}\right)}^{1,0 *} M \otimes N_{\pi_{0}\left(e_{0}\right)}^{1,0} M\right), \quad \bmod \left(\omega_{0}^{N+1}\right),
$$

which is a well-defined tensor, and is called the CR second fundamental form of $M$.

We remark that the notion of $I I_{M}$ in Definition 4 was introduced in a paper by S.H. Wang [Wa06].

Pulling back a lift $\quad$ Let $M \subset \partial \mathbb{H}^{N+1}$ be as above with a point $Q_{0} \in M$. Let $A \in S U(N+$ $1,1), A^{\star} \in \operatorname{Aut}\left(\partial \mathbb{H}^{N+1}\right)$ with $A^{\star}\left(Q_{0}\right)=P_{0}$ and $\widetilde{M}=A^{\star}(M)$. Let $\widetilde{s}: \widetilde{M} \rightarrow S U(N+1,1)$ be a lift. We claim:

$$
s:=A^{-1} \cdot \widetilde{s} \circ A^{\star},
$$

is also a lift from $M$ into $S U(N+1,1)$. Similarly as in (39) and (40), we have

$$
\omega=\left(A^{\star}\right)^{*} \widetilde{\omega}
$$

and

$$
q_{\alpha \beta}^{\mu}=\widetilde{q}_{\alpha \beta}^{\mu} \circ A^{\star} .
$$

where $\omega=s^{*} \Omega, \widetilde{\omega}=\widetilde{s}^{*} \Omega$ and $\Omega$ is the Maurer-Cartan form over $S U(N+1,1)$.

[Example] Consider the maps in (11) and (2):

$$
\begin{aligned}
& \sigma_{p}^{0}(z, w)=\left(z+z_{0}, w+w_{0}+2 i\left\langle z, \overline{z_{0}}\right\rangle\right), \\
& \tau_{p}^{F}\left(z^{*}, w^{*}\right)=\left(z^{*}-\tilde{f}\left(z_{0}, w_{0}\right), w^{*}-\overline{g\left(z_{0}, w_{0}\right)}-2 i\left\langle z^{*}, \overline{\widetilde{f}\left(z_{0}, w_{0}\right)}\right\rangle\right)
\end{aligned}
$$

where $p=\left(z_{0}, w_{0}\right), z=\mathbb{C}^{n}, w=z_{n+1}, \sigma_{p}^{0} \in \operatorname{Aut}\left(\partial \mathbb{H}^{n+1}\right)$, and $\tau_{p}^{F} \in \operatorname{Aut}\left(\partial \mathbb{H}^{N+1}\right)$.

By (19) and (21), these two maps correspond to two matrices:

$$
A_{\sigma_{p}^{0}}=\left[\begin{array}{ccccc}
1 & 0 & \ldots & 0 & 0 \\
z_{01} & 1 & \ldots & 0 & 0 \\
\vdots & \vdots & \ddots & \vdots & \vdots \\
z_{0 n} & 0 & \ldots & 1 & 0 \\
w_{0} & 2 i \overline{z_{01}} & \ldots & 2 i \overline{z_{0 n}} & 1
\end{array}\right] \in S U(n+1,1)
$$


and

$$
A_{\sigma_{p}^{F}}=\left[\begin{array}{ccccc}
1 & 0 & \ldots & 0 & 0 \\
-\widetilde{f}_{01} & 1 & \ldots & 0 & 0 \\
\vdots & \vdots & \ddots & \vdots & \vdots \\
-\widetilde{f}_{0 N-n} & 0 & \ldots & 1 & 0 \\
-\overline{g\left(z_{0}, w\right)} & -2 i \widetilde{f}_{1}\left(z_{0}, w_{0}\right) & \ldots & -2 i \widetilde{f}_{N-n}\left(z_{0}, w_{0}\right) & 1
\end{array}\right] \in S U(N+1,1)
$$

where $z_{0}=\left(z_{01}, \ldots, z_{0 n}\right)$ and $w_{0}=z_{0 n+1}$.

[Example] Consider the map $F_{\lambda, r, \vec{a}, U}=(f, g) \in A u t_{0}\left(\partial \mathbb{H}^{n+1}\right)$

$$
f(z)=\frac{\lambda(z+\vec{a} w) U}{1-2 i\langle z, \vec{a}\rangle-\left(r+i\|\vec{a}\|^{2}\right) w}, g(z)=\frac{\lambda^{2} w}{1-2 i\langle z, \overline{\vec{a}}\rangle-\left(r+i\|\vec{a}\|^{2}\right) w}
$$

where $\lambda>0, r \in \mathbb{R}, \vec{a} \in \mathbb{C}^{n}$ and $U=\left(u_{\alpha \beta}\right)$ is an $(n-1) \times(n-1)$ unitary matrix. By (19) and (21), its corresponding matrix,

$$
A_{F_{\lambda, r, \vec{a}, U}}=\left[\begin{array}{ccccc}
1 & -2 i \overline{a_{1}} & \ldots & -2 i \overline{a_{n}} & -\left(r+i\|\vec{a}\|^{2}\right) \\
0 & \lambda u_{11} & \ldots & \lambda u_{1 n} & \lambda a_{1} \\
\vdots & \vdots & \ddots & \vdots & \vdots \\
0 & \lambda u_{n 1} & \ldots & \lambda u_{n n} & \lambda a_{n} \\
0 & 0 & \ldots & 0 & \lambda^{2}
\end{array}\right]
$$

is not in $S U(n+1,1)$ in general. In fact, we can write

$$
F_{\lambda, r, \vec{a}, U}=F_{\lambda, 0,0, I d} \circ F_{1,0,0, U} \circ F_{1, r, \vec{a}, I d} .
$$

or $A_{F_{\lambda, r, \vec{a}, U}}=A_{F_{\lambda, 0,0, I d}} \cdot A_{F_{1,0,0, U}} \cdot A_{F_{1, r, \vec{a}, I d}}$. Here $A_{F_{1,0,0, U}}$ and $A_{F_{1, r, \vec{a}, I d}}$ are in $S U(N+1,1)$; while $A_{F_{\lambda, 0,0, I d}}$ is in $S U(N+1,1)$ if and only if $\lambda=1$. Therefore

$$
A_{F_{\lambda, r, a, U}} \text { is in } S U(n+1,1) \text { if and only if } \lambda=1 \text {. }
$$

\section{Existence of First-order Adapted Lifts from $M$ into $S U(N+1,1)$ or into $G L^{Q}\left(\mathbb{C}^{N+2}\right)$}

Existence of first-order adapted lifts. Let $\left(M^{\prime}, 0\right)$ be a germ of smooth real hypersurface in $\mathbb{C}^{n+1}$ defined by the defining function

$$
r=\sum_{j=1}^{n} z_{j} \bar{z}_{j}+\frac{i}{2}(w-\bar{w})+o(2) .
$$


We take

$$
\left.\theta=i \partial r=i\left(\sum_{j=1}^{n} \overline{z_{j}} d z_{j}-\frac{1}{2} d w\right)\right)+o(1) .
$$

as a contact form of $M^{\prime}$.

Write $w=u+i v$. Here $v=\sum_{j=1}^{n}\left|z_{j}\right|^{2}+o(2)$. Take $\left(z_{j}, u\right)$ as a coordinates system of $M^{\prime}$. By considering the coordinate map: $h: \mathbb{C}^{n} \times \mathbb{R} \rightarrow M^{\prime},\left(z_{j}, u\right) \mapsto\left(z_{j}, u+i|z|^{2}+o(2)\right)$, we get the pushforward

$$
h_{*}\left(\frac{\partial}{\partial z_{j}}\right)=L_{j}:=\frac{\partial}{\partial z_{j}}+i\left(\overline{z_{j}}+o(1)\right) \frac{\partial}{\partial u}, \quad h_{*}\left(\frac{\partial}{\partial u}\right)=R_{M^{\prime}}:=(1+o(1)) \frac{\partial}{\partial u}
$$

for $j=1,2, \ldots, n$. Then $\left\{L_{j}\right\}_{1 \leq j \leq n}$ form a basis of the complex tangent bundle $T^{1,0} M^{\prime}$ of $M^{\prime}$. Since $d \alpha=-i \sum_{j=1}^{n} d z_{j} \wedge d \overline{z_{j}}$, we see that $R$ is the Reeb vector field of $M^{\prime}$. In particular, as the restriction at 0 , we have

$$
\left.L_{j}\right|_{0}=\left.\frac{\partial}{\partial z_{j}}\right|_{0},\left.\quad R_{M^{\prime}}\right|_{0}=\left.\frac{\partial}{\partial u}\right|_{0} .
$$

Theorem 7.1 Let $M \hookrightarrow \partial \mathbb{H}^{N+1}$ be the image of $H: M^{\prime} \rightarrow \partial \mathbb{H}^{N+1}$ where $M^{\prime} \subset \mathbb{C}^{n+1}$ is a smooth strictly pseudoconvex CR-hypersurface. Then for any point in $M$, the first-order adapted lift $E=\left(E_{0}, E_{\alpha}, E_{\mu}, E_{N+1}\right)$ of $M$ into $S U(N+1,1)$ (hence into $G L^{Q}\left(\mathbb{C}^{N+2}\right)$ ) exists in some neighborhood of the point in $M$.

Proof: Step 1. Without of loss of generality, we assume that $0 \in M$ so that it suffices to construct a lift $E=\left(E_{0}, E_{\alpha}, E_{\mu}, E_{N+1}\right)$ in a neighborhood of the point 0 . Here we denote $[1: 0: \ldots: 0]$ by 0 .

Assume that $M^{\prime}$ is defined by the equation $\operatorname{Im} w=|z|^{2}+o\left(|z|^{2}\right)$ in $(z, w) \in \mathbb{C}^{n} \times \mathbb{C}$ where $w=u+i v$. Assume that $H=\left(1, f_{\alpha}, \phi_{\mu}, g\right)$ is the smooth CR embedding of $M^{\prime}$ into $\partial \mathbb{H}^{N+1}$ with $H(0)=0$ and

$$
f=z+O\left(|(z, w)|^{2}\right), \phi=O\left(|(z, w)|^{2}\right), g=w+O\left(|(z, w)|^{2}\right) .
$$

Let $L_{\alpha}, \alpha=1,2, \ldots, n$ be a basis of the $\mathrm{CR}$ vector fields and $R$ is the Reeb vector field on $M^{\prime}$. Then as in (59) with (60), we have

$$
\left.L_{\alpha}\right|_{0}=\left.\frac{\partial}{\partial z_{j}}\right|_{0}, \text { and }\left.R\right|_{0}=\left.\frac{\partial}{\partial u}\right|_{0} .
$$


It follows that $\bar{L}_{\alpha} H=0$ as $H$ is a CR map. By the Lewy extension theorem, $H$ extends holomorphically to one side of $M^{\prime}$, denoted by $D$, where $D$ is obtained by attaching the holomorphic discs. By applying the maximum principle and the Hopf lemma to the subharmonic function $\sum\left|f_{\alpha}\right|^{2}+\sum\left|\phi_{\mu}\right|^{2}+\frac{i}{2}(g-\bar{g})$ on $D$, it follows that $\frac{\partial \operatorname{Im} g}{\partial v}(0) \neq 0$. Since $\frac{\partial g}{\partial \bar{w}}=0$ and $\frac{\partial \operatorname{Img} g}{\partial u}(0)=0$, we have $R g(0)=\frac{\partial g}{\partial u}(0)=\frac{\partial \operatorname{Im} g}{\partial v}(0) \neq 0$.

Step 2. Direct construction of $E_{0}, E_{\alpha}$ and $E_{N+1} \quad$ We define

$$
E_{0}:=\left[\begin{array}{c}
1 \\
f_{\alpha}(z, w) \\
\phi_{\mu}(z, w) \\
g(z, w)
\end{array}\right]
$$

which can be regarded as a point in $\partial \mathbb{H}^{N+1}$. Then $\left\langle E_{0}, E_{0}\right\rangle=0$ holds:

$$
\sum f_{\alpha} \bar{f}_{\alpha}+\sum \phi_{\mu} \bar{\phi}_{\mu}+\frac{i}{2}(g-\bar{g})=0, \quad \text { on } M .
$$

Apply the CR vector field $L_{\beta}$ to $E_{0}$, we define

$$
\widetilde{E}_{\beta}=\left(0, L_{\beta} f_{\alpha}, L_{\beta} \phi_{\mu}, L_{\beta} g\right)^{t},
$$

which form the basis of the complex tangent bundle $T_{\pi_{0}\left(E_{0}\right)}^{1,0}(M)$. Then in a neighborhood of 0 in $M$, we have

$$
\operatorname{span}_{\mathbb{C}}\left(E_{0}, \widetilde{E}_{\alpha}\right)=\hat{T}_{\pi_{0}\left(E_{0}\right)}^{(1,0)} M .
$$

Now, we have $\left\langle E_{0}, \widetilde{E}_{\alpha}\right\rangle=0$ by applying $L_{\beta}$ to (63):

$$
\sum \bar{f}_{\alpha} L_{\beta} f_{\alpha}+\sum \bar{\phi}_{\mu} L_{\beta} \phi_{\mu}+\frac{i}{2} L_{\beta} g=0
$$

By the Gram-Schmid orthonormalization procedure, we can obtain, from $\left\{\widetilde{E}_{\beta}\right\}$, an orthonormal set with respect to the usual Hermitian inner product $\langle,\rangle_{0}$; we denote it by $\left\{E_{\beta}\right\}$. By the definition (42), we notice that for any $Z=\left(Z^{0}, Z^{A}, Z^{N+1}\right)$ and $Z^{\prime}=\left(Z^{\prime 0}, Z^{\prime A}, Z^{\prime N+1}\right)$,

$$
\left\langle Z, Z^{\prime}\right\rangle=\left\langle\left(\frac{i}{2} Z^{N+1}, Z^{A},-\frac{i}{2} Z^{0}\right),\left(Z_{0}^{\prime}, Z^{\prime A}, Z^{\prime N+1}\right)\right\rangle_{0}=\left\langle\hat{Z}, Z^{\prime}\right\rangle_{0}
$$

where $\langle,\rangle_{0}$ is the usual Hermitian inner product and $\hat{Z}:=\left(\frac{i}{2} Z^{N+1}, Z^{A},-\frac{i}{2} Z^{0}\right)$. Then we see from (64) that

$$
\left\langle E_{0}, E_{\beta}\right\rangle=\left\langle\left(\frac{i}{2} g, f_{\alpha}, \phi_{\mu},-\frac{i}{2}\right),\left(0, L_{\beta} f_{\alpha}, L_{\beta} \phi_{\mu}, L_{\beta} g\right)\right\rangle_{0}=0 .
$$


Also we observe $\left\langle E_{\alpha}, E_{\beta}\right\rangle=\left\langle E_{\alpha}, E_{\beta}\right\rangle_{0}=\delta_{\alpha \beta}$. Then $\left\langle E_{0}, E_{0}\right\rangle=0,\left\langle E_{0}, E_{\beta}\right\rangle=0$ and $\left\langle E_{\alpha}, E_{\beta}\right\rangle=\delta_{\alpha \beta}$ hold.

Applying the Reeb vector field $R$, we define another vector

$$
\widetilde{E}_{N+1}:=\left(0, R f_{\alpha}, R \phi_{\mu}, R g\right)^{t}
$$

over a neighborhood of 0 in $M$ such that

$$
\operatorname{span}\left(E_{0}, E_{\alpha}, \widetilde{E}_{N+1}\right)=\hat{T}_{\pi_{0}\left(E_{0}\right)} M .
$$

We want to construct

$$
E_{N+1}=A E_{0}+B_{\alpha} E_{\alpha}+C \widetilde{E}_{N+1}
$$

such that

$$
\left\langle E_{N+1}, E_{0}\right\rangle=\frac{i}{2},\left\langle E_{\alpha}, E_{N+1}\right\rangle=0, \text { and }\left\langle E_{N+1}, E_{N+1}\right\rangle=0 .
$$

From $\left\langle E_{N+1}, E_{0}\right\rangle=\frac{i}{2}$, we get $\left\langle A E_{0}+B_{\alpha} E_{\alpha}+C \widetilde{E}_{N+1}, E_{0}\right\rangle=\frac{i}{2}$ so that

$$
C=\frac{i}{2\left\langle\widetilde{E}_{N+1}, E_{0}\right\rangle}
$$

By (61), we notice that

$$
\left.\left\langle\widetilde{E}_{N+1}, E_{0}\right\rangle\right|_{0}=\left.\sum \frac{\partial f_{\alpha}}{\partial u}\right|_{0} \bar{f}_{\alpha}(0)+\left.\sum \frac{\partial \phi_{\mu}}{\partial u}\right|_{0} \bar{\phi}_{\mu}(0)+\left.\frac{i}{2} \frac{\partial g}{\partial u}\right|_{0}
$$

and therefore $\left\langle\widetilde{E}_{N+1}, E_{0}\right\rangle(0)=\frac{i}{2} R g(0) \neq 0$.

From $\left\langle E_{N+1}, E_{\alpha}\right\rangle=0$, we get $\left\langle A E_{0}+B_{\beta} E_{\beta}+C \widetilde{E}_{N+1}, E_{\alpha}\right\rangle=0$ so that

$$
B_{\alpha}=-C \delta_{\beta \alpha}\left\langle\widetilde{E}_{N+1}, E_{\beta}\right\rangle=-C\left\langle\widetilde{E}_{N+1}, E_{\alpha}\right\rangle .
$$

From $\left\langle E_{N+1}, E_{N+1}\right\rangle=0$, we get $\left\langle A E_{0}+B_{\beta} E_{\beta}+C \widetilde{E}_{N+1}, A E_{0}+B_{\beta} E_{\beta}+C \widetilde{E}_{N+1}\right\rangle=0$. Since $C\left\langle\widetilde{E}_{N+1}, E_{0}\right\rangle=\frac{i}{2}, \bar{C}\left\langle E_{0}, \widetilde{E}_{N+1}\right\rangle=-\frac{i}{2}, B_{\alpha}=-C\left\langle\widetilde{E}_{N+1}, E_{\alpha}\right\rangle$ and $\overline{B_{\alpha}}=-\bar{C}\left\langle E_{\alpha}, \widetilde{E}_{N+1}\right\rangle$ by (66) and (67), we obtain

$$
-\frac{i}{2} A+\frac{i}{2} \bar{A}-\sum_{\alpha}\left|B_{\alpha}\right|^{2}+|C|^{2}\left\langle E_{N+1}, E_{N+1}\right\rangle=0,
$$

so that

$$
\operatorname{Im}(A)=\sum_{\alpha}\left|B_{\alpha}\right|^{2}-|C|^{2}\left\langle E_{N+1}, E_{N+1}\right\rangle
$$


Therefore $E_{N+1}$ is determined.

So far we have $\left\langle E_{0}, E_{0}\right\rangle=\left\langle E_{N+1}, E_{N+1}\right\rangle=\left\langle E_{0}, E_{\beta}\right\rangle=\left\langle E_{N+1}, E_{\beta}\right\rangle=0,\left\langle E_{\alpha}, E_{\beta}\right\rangle=\delta_{\alpha \beta}$ and $\left\langle E_{0}, E_{N+1}\right\rangle=-\frac{i}{2}$ hold.

Step 3. Construction of $E$ From Step 2, at the point 0, we have vectors

$$
\left.E_{0}\right|_{0}=[1: 0: \ldots: 0],\left.E_{1}\right|_{0}=[0: 1: 0: \ldots: 0], \ldots,\left.E_{n}\right|_{0}=[0: 0: \ldots: 1: 0: \ldots: 0],
$$

and

$$
\left.E_{N+1}\right|_{0}=[0: 0: \ldots: 0: 1] .
$$

Therefore we can define $E$ at the point 0 by

$$
E(0):=I d \in S U(N+1,1) .
$$

For any other point $P$ in a small neighborhood of 0 in $M$, we are going to define $E(P) \in$ $S U(N+1,1)$ as follows.

Write $H(p)=P$ for some $p \in M^{\prime}$. Then we take a map $\Psi_{P} \in S U(N+1,1)$ such that

$$
\Psi_{P}^{\star}(P)=0, \quad T_{0}^{1,0} \Psi(M)=\operatorname{span}_{\mathbb{C}}\left(\left.E_{0}\right|_{0},\left.E_{\alpha}\right|_{0}\right), \quad \text { and } T_{0} \Psi(M)=\operatorname{span}\left(\left.E_{0}\right|_{0},\left.E_{\alpha}\right|_{0},\left.E_{N+1}\right|_{0}\right) .
$$

where $\left.E_{0}\right|_{0},\left.E_{\alpha}\right|_{0}$ and $\left.E_{N+1}\right|_{0}$ are defined in (69) and (70). The map $\Psi_{P}$ can be defined as $A_{F_{1, r, \vec{a}, U}} \circ A_{\sigma_{p}^{F}}$ where $A_{\sigma_{p}^{F}} \in S U(N+1,1)$ as in (54) and $A_{F_{1, r, \vec{a}, U}} \in S U(N+1,1)$ as in (55). Notice in the construction of the normalization $F^{* *}$ and $F^{* * *}$, we can always choose $\lambda=1$ so that (56) can be used. $\Psi_{P}$ is smooth as $P$ varies. Then we define

$$
E(P):=\left(\Psi_{P}^{\star}\right)^{*} E(0)=\left(\Psi_{P}\right)^{-1} E(0) .
$$

This definition is the same as in (50). Since $\Psi_{P}$ is invariant for the Hermitian scalar product $\langle$,$\rangle defined in (42) and E(0)$ satisfies the identities (43), it implies that $E(P)$ satisfies the identities (43), i.e., $E(p) \in S U(N+1,1)$.

As a matrix, we denote $E(P)=\left(\hat{E}_{0}, \hat{E}_{\alpha}, \hat{E}_{\mu}, \hat{E}_{N+1}\right)$. Since the map $\Psi_{P}$ preserves the CR structures and the tangent vector spaces of $M$ and $\Psi_{P}(M)$, we have

$$
\operatorname{span}_{\mathbb{C}}\left(\hat{E}_{0}, \hat{E}_{\alpha}\right)=\left.\operatorname{span}_{\mathbb{C}}\left(E_{0}, E_{\alpha}\right)\right|_{P}, \operatorname{span}\left(\hat{E}_{0}, \hat{E}_{\alpha}, \hat{E}_{N+1}\right)=\left.\operatorname{span}\left(E_{0}, E_{\alpha}, E_{N+1}\right)\right|_{P} .
$$

where $E_{0}, E_{\alpha}$ and $E_{N+1}$ are constructed in Step 2. We remark that we can replace $\left(\hat{E}_{0}, \hat{E}_{\alpha}\right.$, $\left.\hat{E}_{N+1}\right)$ by $\left(E_{0}, E_{\alpha}, E_{N+1}\right)$.

Existence of a more special first-order adapted lifts when $M$ is spherical When $M=F\left(\partial \mathbb{H}^{n+1}\right)$ where $F \in \operatorname{Prop}_{2}\left(\mathbb{H}^{n+1}, \mathbb{H}^{N+1}\right)$, we can construct a more special first-order adapted lift of $M$ into $S U(N+1,1)$ as follows (cf. [HJY09]). 
Let $F=(f, \phi, g) \in \operatorname{Prop}_{2}\left(\partial \mathbb{H}^{n+1}, \partial \mathbb{H}^{N+1}\right)$ be any map with $F=F_{p}^{* * *}$. Then $F(0)=0$. We introduce a local biholomorphic map near the origin

$$
F_{f g}:=(f, g): \mathbb{C}^{n+1} \rightarrow \mathbb{C}^{n+1},\left(z, z_{N+1}\right) \mapsto(f, g)=\left(\hat{z}, \hat{z}_{N+1}\right)
$$

with its inverse

$$
F_{f g}^{-1}: \mathbb{C}^{n+1} \rightarrow \mathbb{C}^{n+1},\left(\hat{z}, \hat{z}_{N+1}\right) \mapsto\left(\left(F_{f g}^{-1}\right)^{(1)}, \ldots,\left(F_{f g}^{-1}\right)^{(n)},\left(F_{f g}^{-1}\right)^{(N+1)}\right)=\left(z, z_{N+1}\right) .
$$

Here we use $\left(\hat{z}, \hat{z}_{N+1}\right)$ as a coordinates system of $M=F\left(\partial \mathbb{H}^{n+1}\right)$ near $F(0)=0$. Denote $\operatorname{Proj}_{f g}: \mathbb{C}^{N+1} \rightarrow \mathbb{C}^{n+1},\left(\hat{z}, \hat{z}_{\mu}, \hat{z}_{N+1}\right) \mapsto\left(\hat{z}, \hat{z}_{N+1}\right)$. Then we have $\operatorname{Proj}_{f g} \circ F=F_{f g}$ :

$$
\begin{array}{ccc}
F: \partial \mathbb{H}^{n+1} & \rightarrow & M \\
& \searrow F_{f g} & \downarrow \operatorname{Proj}_{f g} \\
& & \mathbb{C}^{n+1}
\end{array}
$$

We also have a pair of inverse maps $F: \partial \mathbb{H}^{n+1} \rightarrow M$ and $\left(F_{f g}^{-1}\right) \circ \operatorname{Proj}_{f g}: M \rightarrow \partial \mathbb{H}^{n+1}$.

Locally we can regard $M$ as a graph: $F \circ F_{f g}^{-1}: \mathbb{C}^{n+1} \rightarrow M \subset \mathbb{C}^{N+2}$ :

$$
\left(\hat{z}, \hat{z}_{N+1}\right) \mapsto\left(\hat{z}, \phi\left(\left(F_{f g}\right)^{-1}\left(\hat{z}, \hat{z}_{N+1}\right)\right), \quad \hat{z}_{N+1}\right)
$$

Now let us define a lift of $M$ into $S U(N+1,1)$

$$
e=\left(e_{0}, e_{\alpha}, e_{\mu}, e_{N+1}\right) \in S U(N+1,1), \quad 1 \leq \alpha \leq n, n+1 \leq \mu \leq N
$$

as follows.

We define $e_{0}: M \hookrightarrow \mathbb{C}^{N+2}$ be the inclusion:

$$
e_{0}\left(\hat{z}, \hat{z}_{N+1}\right)=F \circ F_{f g}^{-1}\left(\hat{z}, \hat{z}_{N+1}\right)=\left[1: \hat{z}: \phi\left(\left(F_{f g}\right)^{-1}\left(\hat{z}, \hat{z}_{N+1}\right)\right): \hat{z}_{N+1}\right]^{t}
$$

$\forall\left(\hat{z}, \hat{z}_{N+1}\right) \in \mathbb{C}^{n+1}$. We define $e_{\alpha}: M \rightarrow \mathbb{C}^{N+2}$ for $1 \leq \alpha \leq n$ :

$$
e_{\alpha}:=\frac{1}{\sqrt{\left|L_{\alpha} f\right|^{2}+\left|L_{\alpha} \phi\right|^{2}}}\left[0: L_{\alpha} f: L_{\alpha} \phi: L_{\alpha} g\right]^{t} \circ F_{f g}^{-1} .
$$

where $L_{\alpha}=\frac{\partial}{\partial z^{\alpha}}+2 i \bar{z}^{\alpha} \frac{\partial}{\partial z^{N+1}}$. By the definition (42), we have $\left\langle e_{0}, e_{0}\right\rangle=0$ because $f \cdot \bar{f}+\phi$. $\bar{\phi}-\frac{1}{2 i}(g-\bar{g})=\hat{z} \cdot \bar{z}+\phi\left(\left(F_{f g}\right)^{-1}\left(\hat{z}, \hat{z}_{N+1}\right)\right) \overline{\phi\left(\left(F_{f g}\right)^{-1}\left(\hat{z}, \hat{z}_{N+1}\right)\right)}+\frac{i}{2}\left(\hat{z}_{N+1}-\overline{\hat{z}_{N+1}}\right)=0$ holds on $\partial \mathbb{H}^{n+1}$, and $\left\langle e_{0}, e_{\alpha}\right\rangle=0$ because $L_{\alpha} f \cdot \bar{f}+L_{\alpha} \phi \cdot \bar{\phi}+\frac{i}{2} L_{\alpha} g=0$ holds on $\partial \mathbb{H}^{n+1}$, and $\left\langle e_{\alpha}, e_{\beta}\right\rangle=\delta_{\alpha \beta}$ because $L_{\alpha} f \cdot \overline{L_{\beta} f}+L_{\alpha} \phi \cdot \overline{L_{\beta} \phi}=0$ holds on $\partial \mathbb{H}^{n+1}$ for $\alpha \neq \beta$. 
If we define $\widetilde{e}_{N+1}:=(0, T f, T \phi, T g)^{t} \circ F_{f g}^{-1}$, where $T=\frac{\partial}{\partial u}$ with $z^{N+1}=u+i v$, then $\operatorname{span}\left(e_{0}, e_{\alpha}, \overline{e_{\alpha}}, \widetilde{e}_{N+1}\right)=\hat{T}_{\pi_{0}\left(e_{0}\right)} M$. We then find coefficient functions $A, B_{\alpha}$ and $C$ such that $e_{N+1}=A e_{0}+\sum B_{\alpha} e_{\alpha}+C \widetilde{e}_{N+1}$ satisfies

$$
\left\langle e_{0}, e_{N+1}\right\rangle=-\frac{i}{2},\left\langle e_{\alpha}, e_{N+1}\right\rangle=0,\left\langle e_{N+1}, e_{N+1}\right\rangle=0 .
$$

\section{Relationship among four definitions of $I I_{M}$}

Lemma 8.1 Let $H: M^{\prime} \rightarrow \partial \mathbb{H}^{N+1}$ be a CR smooth embedding where $M^{\prime}$ is a strictly pseudoconvex smooth real hypersurface in $\mathbb{C}^{n+1}$. We denote $M=H\left(M^{\prime}\right)$. Then the following statements are equivalent:

(i) The CR second fundamental form $I I_{M}$ by Definition 1 identically vanishes.

(ii) The CR second fundamental form $I I_{M}$ by Definition 2 identically vanishes.

(iii) The $C R$ second fundamental form $I I_{M}$ by Definition 3 identically vanishes.

(iv) The CR second fundamental form II $I_{M}$ by Definition 4 identically vanishes.

Proof (i) $\Longleftrightarrow$ (ii) by (15).

(iii) $\Longleftrightarrow$ (iv) The equivalence follows by the facts that, for Definition 3 and $4, I I_{M}^{e} \equiv 0$ for one first-order adapted lift $e$ if and only if $I I_{M}^{s} \equiv 0$ for any first-order adapted lift $s$, that a first-order adapted lift from $M$ to $S U(N+1,1)$ must be a first-order adapted lift from $M$ to $G L^{Q}\left(\mathbb{C}^{N+2}\right)$.

(iv) $\Longrightarrow$ (i): Let $M \subset \partial \mathbb{H}^{N+1}$ be a $(2 n+1)$ dimensional $\mathrm{CR}$ submanifold with CR dimension $n$ that admits a first-order adapted lift $e$ into $S U(N+1,1)$. Consider the pullbacked Maurer-Cartan form over $M$ by $e$

$$
\omega=\left(\begin{array}{cccc}
\omega_{0}^{0} & \omega_{\beta}^{0} & \omega_{\nu}^{0} & \omega_{N+1}^{0} \\
\omega_{0}^{\alpha} & \omega_{\beta}^{\alpha} & \omega_{\nu}^{\alpha} & \omega_{N+1}^{\alpha} \\
0 & \omega_{\beta}^{\mu} & \omega_{\nu}^{\mu} & \omega_{N+1}^{\mu} \\
\omega_{0}^{N+1} & \omega_{\beta}^{N+1} & 0 & \omega_{N+1}^{N+1}
\end{array}\right) .
$$

with

$$
\begin{aligned}
& \omega_{0}^{0}+\overline{\omega_{N+1}^{N+1}}=0, \omega_{0}^{N+1}=\overline{\omega_{0}^{N+1}}, \omega_{N+1}^{0}=\overline{\omega_{N+1}^{0}}, \\
& \omega_{A}^{N+1}=2 i \overline{\omega_{0}^{A}}, \omega_{N+1}^{A}=-\frac{i}{2} \overline{\omega_{A}^{0}}, \omega_{B}^{A}+\overline{\omega_{A}^{B}}=0, \omega_{0}^{0}+\omega_{A}^{A}+\omega_{N+1}^{N+1}=0,
\end{aligned}
$$

where $1 \leq A \leq N$. 
Let $\theta=\omega_{0}^{N+1}$ which is a real 1-form by (77). By $d \omega=-\omega \wedge \omega$ and (77), we obtain

$$
d \theta=-\omega_{0}^{N+1} \wedge \omega_{0}^{0}-\omega_{\alpha}^{N+1} \wedge \omega_{0}^{\alpha}-\omega_{N+1}^{N+1} \wedge \omega_{0}^{N+1}=2 i \omega_{0}^{\alpha} \wedge \overline{\omega_{0}^{\alpha}}-\theta \wedge\left(\omega_{0}^{0}+\overline{\omega_{0}^{0}}\right)=i \theta^{\alpha} \wedge \overline{\theta^{\alpha}},
$$

where we denote

$$
\theta^{\alpha}=\sqrt{2} \omega_{0}^{\alpha}+c_{\alpha} \theta
$$

for some functions $c_{\alpha}$. Therefore, (8) holds and hence $M$ is a strictly pseudoconvex pseudohermitian manifold with an admissible coframe $\left(\theta, \theta^{\alpha}\right)$. Hence Definition 4 of $I I_{M} \equiv 0$ implies Definition 1 of $I I_{M} \equiv 0$.

(i) $\Longrightarrow$ (iv): Definition 1 of $I I_{M}$ gives a coframe $\left(\theta, \theta^{\alpha}\right)$ which corresponds to Definition 2 of $I I_{M}$ with respect to a defining function $\rho$ of $M$ in $\partial \mathbb{H}^{N+1}$.

Now take a first-order adapted lift $e$ from $M$ into $S U(N+1,1)$. By (78), it corresponds to a coframe $\left(\theta, \theta^{\alpha}\right)$ on $M$ and by (16), it corresponds Definition 2 of $I I_{M}$ by some choice of the defining function $\hat{\rho}$ of $M$ in $\partial \mathbb{H}^{N+1}$.

The above $\rho$ and $\hat{\rho}$ may not be the same. But Definition 2 of $I I_{M} \equiv 0$ is independent of choice of defining functions, which gives (i) $\Longrightarrow$ (iv).

\section{$9 \quad$ Proof of Theorem 1.1}

Lemma 9.1 (cf. [EHZ04], corollary 5.5) Let $H: M^{\prime} \rightarrow M \hookrightarrow \partial \mathbb{H}^{N+1}$ be a smooth $C R$ embedding of a strictly pseudoconvex smooth real hypersurface $M \subset \mathbb{C}^{n+1}$. Denote by $\left(\omega_{\alpha}^{\mu}{ }_{\beta}\right)$ the $C R$ second fundamental form matrix of $H$ relative to an admissible coframe $\left(\theta, \theta^{A}\right)$ on $\partial \mathbb{H}^{N+1}$ adapted to $M$. If $\omega_{\alpha \beta}^{\mu} \equiv 0$ for all $\alpha, \beta$ and $\mu$, then $M^{\prime}$ is locally $C R$-equivalent to $\partial \mathbb{H}^{n+1}$.

Proof of Theorem 1.1 Step 1. Reduction to a problem for geometric rank By Lemma 8.1 and Lemma 9.1 and the hypothesis that the CR second fundamental form identically vanishes, we know that $M$ is locally $\mathrm{CR}$ equivalent to $\partial \mathbb{H}^{n+1}$.

Then $M$ is the image of a local smooth CR map $F: U \subset \partial \mathbb{H}^{n+1} \rightarrow M \subset \partial \mathbb{H}^{N+1}$ where $U$ is a open set in $\partial \mathbb{H}^{n+1}$. By a result of Forstneric [Fo89], the map $F$ must be a rational map. It suffices to prove that $F$ is equivalent to a linear map. By Lemma 2.2, it is sufficient to prove that the geometric rank of $F$ is zero: $\kappa_{0}=0$.

Suppose $\kappa_{0}>0$ and we seek a contradiction.

Step 2. Reduction to a lift of $\left(\left(H \circ \tau_{p}^{F}\right)(M), 0\right) \quad$ Take any point $p \in U \subset \partial \mathbb{H}^{n+1}$ with $\kappa_{0}=\kappa_{0}(p)>0$, and consider the associated map (see Lemma 2.1)

$$
F_{p}^{* * *}=H \circ \tau_{p}^{F} \circ F \circ \sigma_{p}^{0} \circ G: \partial \mathbb{H}^{n+1} \rightarrow \partial \mathbb{H}^{N+1}, \quad F_{p}^{* * *}(0)=0,
$$


where $\sigma_{p}^{0}$ is defined in (11), $\tau_{p}^{F}$ is defined in (2), $G \in A u t_{0}\left(\mathbb{H}^{n+1}\right)$ and $H \in A u t_{0}\left(\mathbb{H}^{N+1}\right)$ are automorphisms. By Theorem $2.3, F_{p}^{* * *}=(f, \phi, g)$ satisfies the following normalization conditions:

$$
\left\{\begin{aligned}
f_{j} & =z_{j}+\frac{i \mu_{j}}{2} z_{j} w+o_{w t}(3), \quad \frac{\partial^{2} f_{j}}{\partial w^{2}}(0)=0, j=1 \cdots, \kappa_{0}, \mu_{j}>0, \\
f_{j} & =z_{j}+o_{w t}(3), \quad j=\kappa_{0}+1, \cdots, n-1 \\
g & =w+o_{w t}(4), \\
\phi_{j l}= & \mu_{j l} z_{j} z_{l}+o_{w t}(2), \text { where }(j, l) \in \mathcal{S} \text { with } \mu_{j l}>0 \text { for }(j, l) \in \mathcal{S}_{0} \\
& \text { and } \mu_{j l}=0 \text { otherwise }
\end{aligned}\right.
$$

where $\mu_{j l}=\sqrt{\mu_{j}+\mu_{l}}$ for $j, l \leq \kappa_{0} j \neq l, \mu_{j l}=\sqrt{\mu_{j}}$ if $j \leq \kappa_{0}$ and $l>\kappa_{0}$ or if $j=l \leq \kappa_{0}$. Here the assumption that $\kappa_{0}>0$ is used.

From (79) we obtain

$$
\begin{array}{ccc}
(M, F(p)) & \stackrel{H \circ \tau_{p}^{F}}{\longrightarrow} & \left(\begin{array}{c}
\left.H \circ \tau_{p}^{F}(M), 0\right) \\
\uparrow F_{p}^{* * *}
\end{array}\right. \\
\left(\partial \mathbb{H}^{n+1}, p\right) & \stackrel{\sigma_{p}^{0} \circ G}{\longleftarrow} & \left(\partial \mathbb{H}^{n+1}, 0\right)
\end{array}
$$

If we can show that there exists a first-order adapted lift $e$ from the submanifold $H \circ \tau_{p}^{F}(M)$ near 0 into $S U(N+1,1)$ such that the corresponding $\mathrm{CR}$ second fundamental form

$$
I I_{H \circ \tau_{p}^{F}(M)}^{e} \neq 0 \text { at } 0
$$

then we obtain a first-order adapted lift $\widetilde{e}:=\left(H \circ \tau_{p}^{F}\right)^{-1} \circ e \circ H \circ \tau_{p}^{F}$ from the submanifold $M$ near $F(p)$ into $G L^{Q}\left(\mathbb{C}^{N+1}\right)$ such that the corresponding $\mathrm{CR}$ second fundamental form

$$
I I_{M}^{\widetilde{e}} \neq 0 \text { at } F(p) .
$$

Notice that the map $H \circ \tau_{p}^{F} \in G L^{Q}\left(\mathbb{C}^{N+2}\right)$ but $H \circ \tau_{p}^{F} \notin S U(N+1,1)$, so that the lift $\widetilde{e}$ is not from $M$ into $S U(N+1,1)$. This is why we have to introduce Definition 3.

Since we take arbitrary $p \in \partial \mathbb{H}^{n+1}$, from (82) it concludes that $I I_{M} \not \equiv 0$, but this is a desired contradiction.

Step 3. Calculation of the second fundamental form It remains to prove existence of the lift $e$ such that (81) holds.

The lift $e$ constructed in the second half of Section 7 is a first-order adapted lift from $H \circ \tau_{p}^{F}(M)$ near 0 into $S U(N+1,1)$ which defines a $\mathrm{CR}$ second fundamental form as a 
tensor $I I_{H \circ \tau_{p}^{F}(M)}^{e}=q_{\alpha \beta}^{\mu} \omega^{\alpha} \omega^{\beta} \otimes\left(\underline{\left.e_{\mu}\right)}\right.$ in (49). If we can show

$$
q_{\alpha \beta}^{\mu}(0)=\left.\frac{\partial^{2} \phi_{\mu}}{\partial z_{\alpha} \partial z_{\beta}}\right|_{0}
$$

where $F_{p}^{* * *}=(f, \phi, g)=\left(f_{\alpha}, \phi_{\mu}, g\right)$. Since we assume that $\kappa_{0}>0$, by (80) and (83), it implies $q_{\alpha \beta}^{\mu}(0) \neq 0, \forall \alpha, \beta$ and $\mu$, i.e., $I I_{H \circ \tau_{p}^{F}(M)}^{e} \neq 0$. This proves (81).

Let $E=\left(e_{0}, e_{\alpha}, \hat{E}_{\mu}, e_{N+1}\right)$ be the lift constructed in Theorem 7.1 (see the remark at the end of the proof of Theorem (7.1) and in (74) (75) and (76). Since $\left.E\right|_{0}=I d$, we have

$$
\left.\omega\right|_{0}=\left.\left(\left.E^{-1}\right|_{0}\right)(d E)\right|_{0}=\left.d E\right|_{0}
$$

so that

$$
\left.\omega\right|_{0}=\left.\left[\begin{array}{cccc}
0 & * & \ldots & * \\
d z_{1} & * & \ldots & * \\
\vdots & \vdots & & \vdots \\
d z_{n} & * & \ldots & * \\
* & * & \ldots & * \\
\vdots & \vdots & & \vdots \\
* & * & \ldots & * \\
d w & * & \ldots & *
\end{array}\right]\right|_{0}
$$

Hence $\left.\omega_{0}^{1}\right|_{0}=d z_{1}, \ldots,\left.\omega_{0}^{n}\right|_{0}=d z_{n},\left.\omega_{0}^{N+1}\right|_{0}=d z_{N+1}$. Then by applying the chain rule, we obtain

$$
\left.\omega_{j}^{\mu}\right|_{0}=\left.d E_{j}^{\mu}\right|_{0}=\left.d\left(\left(L_{j} \phi_{\mu}\right) \circ\left(F_{f g}\right)^{-1}\right)\right|_{0}=\left.\frac{\partial}{\partial z_{k}}\left(\left(L_{j} \phi_{\mu}\right) \circ\left(F_{f g}\right)^{-1}\right)\right|_{0} d z_{k}=\left.\left.\frac{\partial^{2} \phi_{\mu}}{\partial z_{k} \partial z_{j}}\right|_{0} \omega_{0}^{k}\right|_{0},
$$

for any $j, k \in\{1,2, \ldots, n, N+1\}, n+1 \leq \mu \leq N$. Hence (83) is proved. The proof of Theorem 1.1 is complete.

\section{References}

[CM74] S. S. Chern and J. K. Moser, Real hypersurfaces in complex manifolds. Acta Math. 133 (1974), 219-271.

[EHZ04] P. Ebenfelt, X. Huang and D. Zaitsev, Rigidity of CR-immersions into spheres. Comm. Anal. Geom. 12(2004), no. 3, 631-670. 
[Fa88] J. Faran, The nonembeddability of real hypersurfaces in sphere, Proc. A.M.S. 103(1988), 902-904.

[Fa90] J. Faran, A reflection principle for proper holomorphic mappings and geometric invariants, Math. Z. 203 (1990), 363-377.

[F086] F. Forstneric, Embedding strictly pseudoconvex domains into balls, Trans. A.M.S. 295(1986), 347-368.

[KO06] S.Y. Kim and J.W. Oh, Local embeddability of pseudohermitian manifolds into spheres. Math. Ann. 334 (2006), no. 4, 783-807.

[Fo89] F. Forstneric, Extending proper holomorphic mappings of positive codimension, Invent. Math., 95 (1989), 31-62.

[Hu99] X. Huang, On a linearity problem of proper holomorphic mappings between balls in complex spaces of different dimensions, J. of Diff. Geom. 51(1999), 13-33.

[Hu03] X. Huang, On a semi-rigidity property for holomorphic maps, Asian J. Math. Vol(7) No. 4(2003), 463-492.

[HJY09] X. Huang, S. Ji and W. Yin, The third gap for proper holomorphic maps between balls, preprint.

[IL03] T.A. Ivey and J.M. Landsberg, Cartan for beginners: differential geometry via moving frames and exterior differential systems. Graduate Studies in Mathematics, 61. American Mathematical Society, Providence, RI, 2003. xiv+378 pp.

[La01] B. Lamel, A reflection principle for real-analytic submanifolds of complex spaces, J. Geom. Anal. 11, no. 4, 625-631, (2001).

[T75] N. Tanaka, A differential geometric study on strongly pseudo-convex manifolds. Lectures in Mathematics, Department of Mathematics, Kyoto University, No. 9. Kinokuniya Book-Store Co., Ltd., Tokyo, 1975.

[Wa06] S.H. Wang, A gap rigidity for proper holomorphic maps from $\mathbb{B}^{n+1}$ to $\mathbb{B}^{3 n-1}$. arXiv.math/0604382v1 [math.DC], 2006.

[We78] S.M. Webster Pseudo-Hermitian structures on a real hypersurface. J. Differential Geom. 13 (1978), no. 1, 25-41. 
[We79] S.M. Webster The rigidity of $C$ - $R$ hypersurfaces in a sphere. Indiana Univ. Math. J. 28 (1979), no. 3, 405-416.

[Za08] D. Zaitsev, Obstructions to embeddability into hyperquadrtics and explicit examples. Math Ann, 342(2088), 695-726.

Shanyu Ji (shanyuji@math.uh.edu), Department of Mathematics, University of Houston, Houston, TX 77204;

Yuan Yuan (yuanyuan@math.rutgers.edu), Department of Mathematics, Rutgers University, Piscataway, NJ 08854. 